\title{
MOTION OF A DROPLET FOR THE STOCHASTIC MASS-CONSERVING ALLEN-CAHN EQUATION
}

\author{
D.C. ANTONOPOULOU ${ }^{\$ *}$, P.W. BATES ${ }^{\ddagger}$, D. BLÖMKER $\sharp$, G.D. KARALI ${ }^{\dagger *}$
}

\begin{abstract}
We study the stochastic mass-conserving Allen-Cahn equation posed on a smoothly bounded domain of $\mathbb{R}^{2}$ with additive, spatially smooth, space-time noise. This equation describes the stochastic motion of a small almost semicircular droplet attached to domain's boundary and moving towards a point of locally maximum curvature. We apply Itô calculus to derive the stochastic dynamics of the center of the droplet by utilizing the approximately invariant manifold introduced by Alikakos, Chen and Fusco [2] for the deterministic problem. In the stochastic case depending on the scaling, the motion is driven by the change in the curvature of the boundary and the stochastic forcing. Moreover, under the assumption of a sufficiently small noise strength, we establish stochastic stability of a neighborhood of the manifold of boundary droplet states in the $L^{2}$ - and $H^{1}$-norms, which means that with overwhelming probability the solution stays close to the manifold for very long time-scales.
\end{abstract}

Keywords: Stochastic Allen-Cahn, mass conservation, droplet motion, additive noise, invariant manifold, stochastic dynamics, stochastic stability, Itô calculus.

\section{INTRODUCTION}

1.1. The problem. We consider the initial and boundary value problem for the mass conserving AllenCahn equation posed on a two-dimensional bounded smooth domain $\Omega$, and introduce an additive spatially smooth and white in time space-time noise $\dot{V}$

$$
\begin{aligned}
& \partial_{t} \phi^{\hat{\varepsilon}}(y, t)=\hat{\varepsilon}^{2} \Delta_{y} \phi^{\hat{\varepsilon}}(y, t)-f\left(\phi^{\hat{\varepsilon}}(y, t)\right)+\frac{1}{|\Omega|} \int_{\Omega} f\left(\phi^{\hat{\varepsilon}}(y, t)\right) d y+\dot{V}(y, t), \quad y \in \Omega, \quad t>0, \\
& \partial_{n} \phi^{\hat{\varepsilon}}(y, t)=0, \quad y \in \partial \Omega, \quad t>0, \\
& \phi^{\hat{\varepsilon}}(y, 0)=\phi_{0}^{\hat{\varepsilon}}(y), \quad y \in \Omega .
\end{aligned}
$$

Here, $\hat{\varepsilon}$ is a small positive parameter, $\Omega \subset \mathbb{R}^{2}$ of area $|\Omega|$ is a bounded domain with sufficiently smooth boundary $\partial \Omega$, and $\partial_{n}$ is the exterior normal derivative to $\partial \Omega$. The function $f$ is the derivative of a doublewell potential, which we denote by $F$. We assume that $f$ is smooth, $f( \pm 1)=0<f^{\prime}( \pm 1)$ and $f$ has exactly one other zero that lies in $(-1,1)$. The standard example is $f(u):=u^{3}-u$ which will be considered, for simplicity, in the whole presentation although the result holds for more general nonlinearities.

The deterministic problem, i.e., when $\dot{V}=0$, was first studied by J. Rubinstein and P. Sternberg, 33, then by N. Alikakos, X. Chen and G. Fusco, 2, and later by P. Bates and J. Jin in [6]. In [2, the authors analyzed the problem's long-time dynamics and established existence of stable sets of solutions corresponding to the motion of a small, almost semicircular interface (droplet) intersecting the boundary of the domain

1991 Mathematics Subject Classification. 35K55, 35K40, 60H30, 60H15.

P.B. was supported in part by NSF DMS 0908348 and 1413060. P.B. and G.K. were supported in part by the IMA at the

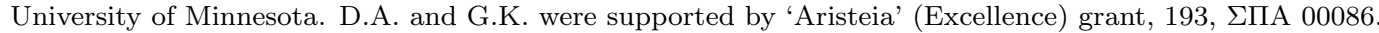

$¥$ Department of Mathematics, Michigan State University, East Lansing, MI 48824, USA.

\# Institut für Mathematik, Universität Augsburg, 86135 Augsburg, Germany.

$\dagger$ Department of Mathematics and Applied Mathematics, University of Crete, GR-714 09 Heraklion, Greece.

\$ Department of Mathematics, University of Chester, Thornton Science Park, CH2 4NU, UK.

* Institute of Applied and Computational Mathematics, FORTH, GR-711 10 Heraklion, Greece. 
and moving towards a point of locally maximal curvature. In [6], the authors proved the existence of a global invariant manifold of droplet states using the approximation given in [2].

The Allen-Cahn equation, also called Model A in the theory of dynamics of critical phenomena (cf. [26]), describes the evolution of the rescaled concentration $\phi^{\hat{\varepsilon}}$ of one species of a two-phase mixture, for example a binary alloy, occupying a bounded domain $\Omega$. The small positive parameter $\hat{\varepsilon}$ represents the surface tension associated with interfacial regions that are generated during phase separation, cf. [3]. The double-well potential $F$ favors layered functions that take values close to its minima \pm 1 . The zero level sets of such a function are called interfaces, while the values close to \pm 1 are called states, close to being a pure state away from the interface. Due to mass conservation and as the average concentration is close to being a pure state, a phase separation begins by nucleation ( $\mathrm{cf}[\mathbf{5}]$ ). Note that in the case of the mass being close to zero, when the two components are roughly equal, as with the Cahn-Hilliard equation (Model B of critical dynamics), the total mass of each component of the mixture is also conserved but separation from a nearly homogeneous state occurs during spinodal decomposition, see [14, 11, 19].

If the states are separated, the total perimeter of the interfaces decreases in time, [20, 21, 13. For the one-dimensional case we refer to [16, 15, 23, 34, 31. See also the classical results of [28, 29] for perimeter minimization of interfaces. For the two-dimensional stationary and stable single layer problem, Chen and M. Kowalczyk in [17] proved that in the limit $\hat{\varepsilon} \rightarrow 0^{+}$this interface becomes a circular arc intersecting the boundary orthogonally and encloses a point on the boundary where the curvature has a local maximum. Alikakos, Chen and Fusco in 2, restricted their analysis to a single connected interface (curve) of shape close to a small semicircular-arc intersecting the outer boundary $\partial \Omega$. This so-called droplet maintains an almost semicircular shape for economizing the perimeter and therefore, as shown in [2, its evolution is fully described in terms of the motion of its center along the outer boundary.

In the absence of the mass-conserving (non-local) term, the multi-dimensional stochastic Allen-Cahn equation driven by a special, non-smooth in time and smooth in space, multiplicative noise was considered in [32. This equation stands as a formal approximation to a mean curvature flow with stochastic forcing. The authors proved that, in the sharp interface $(\varepsilon \rightarrow 0)$ limit, the solutions are tight and converge to phaseindicator functions. The existence and properties of such a stochastic flow combining curvature motion and stochastic perturbations generated by Brownian vector fields were first established by N.K. Yip in 38 in the context of geometric measure theory; physically, these perturbations appear due to thermal fluctuations and possible impurities in the alloy. The analysis of M. Röger and H. Weber in 32, which was based on energy estimates, is related to Yip's construction of an iterative scheme where a sequence of sets with randomly perturbed boundaries was introduced.

The sharp interface limit for the one-dimensional Allen-Cahn equation with an additive space-time white noise, was studied by H. Weber in [36] on the level of invariant measures. Later, in [30, F. Otto, H. Weber and M. Westdickenberg investigated the competition between some energy functional, minimized for small noise strength, and the entropy induced by a large system size. They proved a uniform distribution for the transition layer's positions for scales larger than the logarithm of the inverse noise strength. We also refer to the interesting results of M. Hairer, M. Ryser and H. Weber in [24, where a mollified additive white spacetime noise was introduced into the Allen-Cahn equation and the limiting behavior for correlation length of the noise to 0 of the solution was investigated, which yields very rough noise in the limit. In the limit when both the noise intensity $\sqrt{\varepsilon}$ and the correlation length $\delta$ of the noise go to zero, M. Hairer and H. Weber [25] derived a large deviation principle for solutions defined only via the recently developed theory of regularity structures.

Recently, in 9, N. Berglund and S. Dutercq considered a discretized one-dimensional version of the stochastic mass-conserving Allen-Cahn equation and studied metastable dynamics. The authors showed that the long-term dynamics of this system is similar to Kawasaki-type exchange dynamics and determined explicitly its transition probabilities. 
As in 2] and [6], in the present work we consider a single small droplet and so the average concentration $m \in(-1,1)$ is assumed to satisfy

$$
m=1-\frac{\pi \delta^{2}}{|\Omega|},
$$

for some $0<\delta \ll 1$, while the parameter $\hat{\varepsilon}$ is sufficiently small such that $0<\hat{\varepsilon} \ll \delta^{3}$. Note that in the case of $N$ well separated boundary bubbles of almost equal area, while the bubbles interact most strongly with the boundary to derive their motion and stability (if stationary), they nevertheless interact with each other and $N-1$ extra degrees of instability arise, much as in the LSW theory of coarsening in a supersaturated solution (see [27] and [35).

When $\dot{V} \equiv 0$, if $z\left(\hat{\xi}_{0}\right)$ is a point of $\partial \Omega$ where the curvature has a strict extremum, then there exists a unique equilibrium $\phi(y)$ of 1.1 with zero level set close to the circle of radius $\delta$ centered at this point. Moreover, for layered initial data whose interface is close to the semicircle centered at $z\left(\hat{\xi}_{0}\right)$ of radius $\delta$, the solution of (1.1) is layered also, with interface close to a semicircle of the same radius centered at some point $z(\hat{\xi}(t))$ of $\partial \Omega,[2$.

Note that the mass conservation constraint

$$
\frac{1}{|\Omega|} \int_{\Omega} \phi^{\hat{\varepsilon}}(y, t) d y=m \text { for any } t \geq 0,
$$

holds if and only if

$$
\begin{aligned}
0 & =\partial_{t}\left(\int_{\Omega} \phi^{\hat{\varepsilon}}(y, t) d y\right)=\int_{\Omega} \phi_{t}^{\hat{\varepsilon}}(y, t) d y \\
& =\int_{\Omega} \hat{\varepsilon}^{2} \Delta_{y} \phi^{\hat{\varepsilon}}(y, t) d y-\int_{\Omega} f\left(\phi^{\hat{\varepsilon}}(y, t)\right) d y+\int_{\Omega} \frac{1}{|\Omega|} \int_{\Omega} f\left(\phi^{\hat{\varepsilon}}(y, t)\right) d y d y+\int_{\Omega} \dot{V}(y, t) d y \\
& =\int_{\Omega} \dot{V}(y, t) d y .
\end{aligned}
$$

Hence, mass conservation holds for the stochastic problem only if the spatially smooth additive noise satisfies

$$
\int_{\Omega} \dot{V}(y, t) d y=0 \text { for any } t \geq 0
$$

This means that if a Fourier series expansion is used for the definition of the stochastic forcing, then there is no noise on the constant mode.

Following [2], in order to fix the size of the droplet, we introduce in (1.1) the following change of variables:

$$
y=\delta x, \quad \hat{\varepsilon}=\varepsilon \delta, \quad w^{\varepsilon}(x, t)=\phi^{\hat{\varepsilon}}(y, t), \quad \dot{W}(x, t)=\dot{V}(y, t), \quad \Omega_{\delta}=\delta^{-1} \Omega:=\left\{x \in \mathbb{R}^{2}: \delta x \in \Omega\right\},
$$

and obtain the equivalent problem

$$
\begin{aligned}
& \partial_{t} w^{\varepsilon}(x, t)=\varepsilon^{2} \Delta w^{\varepsilon}(x, t)-f\left(w^{\varepsilon}(x, t)\right)+\frac{1}{\left|\Omega_{\delta}\right|} \int_{\Omega_{\delta}} f\left(w^{\varepsilon}(x, t)\right) d x+\dot{W}(x, t), \quad x \in \Omega_{\delta}, \quad t>0, \\
& \partial_{n} w^{\varepsilon}(x, t)=0, \quad x \in \partial \Omega_{\delta}, \quad t>0 \\
& w^{\varepsilon}(x, 0)=w_{0}^{\varepsilon}(x), \quad x \in \Omega_{\delta} .
\end{aligned}
$$

Here, $\Delta=\Delta_{x}$ is the Laplacian with respect to $x, \dot{W}(x, t)$ is again an additive smooth in space, space-time noise, and $\partial_{n}$ is the normal derivative to $\partial \Omega_{\delta}$.

1.2. Assumptions on the noise. The noise $\dot{W}$ is defined as the formal derivative of a Wiener process depending on $\varepsilon$, which is given by a Fourier series with coefficients being independent Brownian motions in time. The noise $\dot{W}$ arises from a rescaling of $\dot{V}$; the change of variables involves the parameter $\delta>0$ which is considered small but fixed (see Remark 1.1). 
Let $W$ be a $\mathcal{Q}$-Wiener process in the underlying Hilbert space $H:=L^{2}\left(\Omega_{\delta}\right)$, where the covariance operator $\mathcal{Q}$ is a symmetric non-negative operator and $\left(e_{k}\right)_{k \in \mathbb{N}}$ is a complete $L^{2}\left(\Omega_{\delta}\right)$-orthonormal basis of eigenfunctions with corresponding non-negative eigenvalues $a_{k}^{2}$, so that

$$
\mathcal{Q} e_{k}=a_{k}^{2} e_{k} \text {. }
$$

Then, $W$ is given as the Fourier series

$$
W(\cdot, t):=\sum_{k=1}^{\infty} a_{k} \beta_{k}(t) e_{k}(\cdot)=\mathcal{Q}^{1 / 2} \sum_{k=1}^{\infty} \beta_{k}(t) e_{k}(\cdot),
$$

for a sequence of independent real-valued Brownian motions $\left\{\beta_{k}(t)\right\}_{t \geq 0}$. For details see DaPrato and Zabzcyck [18. For the results we always need to assume a noise strength which is small in $\varepsilon$. Thus the covariance $\mathcal{Q}$ and its eigenvalues $a_{k}^{2}$ although not explicitly stated in the notation will depend on $\varepsilon$. Note that, due to rescaling, $a_{k}, \mathcal{Q}$, and $e_{k}$ will depend on $\delta$. We will discuss that below, but suppress this dependence in our notation.

The process $W$ is assumed to satisfy

$$
\int_{\Omega_{\delta}} \dot{W}(x, t) d x=0 \quad \text { for any } \quad t \geq 0,
$$

so that the mass conservation condition 1.2 holds true.

As our approach is based on the application of the Itô-formula, we will always assume that the trace of the operator $\mathcal{Q}$ is finite, i.e.,

$$
\operatorname{trace}(\mathcal{Q}):=\sum_{k=1}^{\infty} a_{k}^{2}=\eta_{0}<\infty
$$

Let $\|\mathcal{Q}\|$ be the induced operator norm in $L^{2}$. We will also measure the noise strength by

$$
\eta_{1}=\|\mathcal{Q}\|=\max \left\{a_{1}^{2}, a_{2}^{2}, a_{3}^{2}, \ldots\right\} .
$$

Observe that

$$
\eta_{1}=\|\mathcal{Q}\| \leq \operatorname{trace}(\mathcal{Q})=\eta_{0} .
$$

The required smoothness in space of the noise affords us another measure of its strength

$$
\eta_{2}:=\sum_{i=1}^{\infty} a_{i}^{2}\left\|\nabla e_{i}\right\|^{2}=\operatorname{trace}(\mathcal{Q} \Delta)<\infty .
$$

This assumption will be used later when the Itô-formula is applied for the proof of certain $H^{1}$-norm estimates.

1.3. Scaling of the noise. Our results depend on the order of magnitude of $\widetilde{\eta}:=\left(\eta_{0}, \eta_{1}, \eta_{2}\right)$ in terms of $\varepsilon$.

We have suppressed the $\varepsilon$-dependence of the noise but allow this dependence on $\varepsilon$ of the $a_{i}$ 's to be quite general. Nevertheless, for our proofs, it is only essential how $\widetilde{\eta}$ tends to zero with $\varepsilon$.

A typical example would be that the noise $\dot{W}$ itself is independent of $\varepsilon$ apart from a global $\varepsilon$-dependent prefactor, which then is the noise strength. In that example all $a_{i}^{2}$ 's and $\eta_{k}$ 's depend on $\varepsilon$ only via a common prefactor.

Remark 1.1. Although we will neglect it later by assuming that $\delta$ is a fixed small constant, let us briefly discuss the $\delta$-dependence of the noise in the simplified case where we have a common $\varepsilon$-dependent prefactor only. Thus, we have before rescaling to $\Omega_{\delta}$

$$
V(y, t)=V(y, t ; \hat{\varepsilon})=\sum \beta_{i}(t) \hat{e}_{i}(y) c_{i}(\hat{\varepsilon}) \text { with } c_{i}(\hat{\varepsilon})=\hat{\varepsilon}^{a} c_{i}
$$

where $c_{i}$ is independent of $\hat{\varepsilon}$, and the $\hat{e_{i}}$ 's form an orthonormal basis in $L^{2}(\Omega)$. Then we apply the change of variables

$$
\hat{\varepsilon}=\varepsilon \delta, \quad \Omega_{\delta}=\delta^{-1} \Omega, \quad y=\delta x,
$$


which leads to the following change of the Wiener processes defining the noise:

$$
\begin{aligned}
W(x, t ; \varepsilon)=V(y, t ; \hat{\varepsilon}) & =\hat{\varepsilon}^{a} \sum \beta_{i}(t) \hat{e}_{i}(y) c_{i} \\
& =\varepsilon^{a} \delta^{a} \sum \beta_{i}(t) \hat{e}_{i}(\delta x) c_{i}=\varepsilon^{a} \delta^{a-1} \sum \beta_{i}(t) e_{i}(x) c_{i},
\end{aligned}
$$

where we defined $e_{i}(x):=\delta \hat{e}_{i}(\delta x)$, which forms an orthonormal basis in $L^{2}\left(\Omega_{\delta}\right)$. Thus, the strength of the noise $\dot{W}$, in the transformed representation, is of order $\varepsilon^{a} \delta^{a-1}$ and the eigenvalues of the covariance are $a_{i}^{2}(\varepsilon, \delta)=\varepsilon^{2 a} \delta^{2 a-2} c_{i}^{2}$.

As already mentioned, in this paper we consider $\delta$ very small but fixed. Our aim is to analyze the asymptotic problem for $0<\varepsilon \ll 1$, as $\varepsilon \rightarrow 0^{+}$. Therefore, we suppress the explicit dependence on $\delta$ in the notation.

1.4. Notation. Throughout this paper we will use the following $\mathcal{O}$-notations.

Definition $1.2(\mathcal{O}$-Notation for scalars). If there exists a universal constant $C>0$ such that

$$
|f| \leq C g \text {, for } 0<g \text { sufficiently small, }
$$

where $f$ and $g$ are scalars, then we call $f$ a scalar of order $\mathcal{O}(g)$.

Definition $1.3\left(\mathcal{O}_{\mathcal{H}}\right.$-Notation for functions). Let $f$ be a function in the normed space $\left(\mathcal{H},\|\cdot\|_{\mathcal{H}}\right)$ and $k \in \mathbb{R}$. We call $f$ a function of order $\mathcal{O}_{\mathcal{H}}\left(\varepsilon^{k}\right)$ if

$$
\|f\|_{\mathcal{H}} \leq C \varepsilon^{k}, \text { for } 0<\varepsilon \text { sufficiently small, }
$$

for some universal constant $C>0$.

The problem addressed is stochastic. Frequently we shall present bounds of some stochastic processes; these bounds are considered on the processes paths usually only up to a stopping time and hold almost surely (a.s.) with respect to probability.

Definition $1.4(\mathcal{O}$-Notation for random processes up to a stopping time). Let $X$ be a random process and $k \in \mathbb{R}$. We call $X$ a process of order $\mathcal{O}\left(\varepsilon^{k}\right)$ up to a stopping time $\tau$, if

$$
\sup _{t \in[0, \tau]}|X| \leq C \varepsilon^{k}, \text { for } 0<\varepsilon \text { sufficiently small, }
$$

for some universal constant $C>0$.

More specifically, if $X$ is a random variable, like the supremum of a stochastic process up to a stopping time, which is defined on a probability space and taking values in $\mathbb{R}$ then the notation $X \leq b$, for $b \in \mathbb{R}$, refers to

$$
\mathbb{P}(X \leq b)=1, \quad \text { or equivalently, } \quad X \leq b \quad \mathbb{P} \text {-a.s. }
$$

In the sequel, we apply certain norms (for example in $L^{q}\left(\Omega_{\delta}\right)$ or in $H^{1}\left(\Omega_{\delta}\right)$ ) to the time-dependent stochastic processes; these norms are then real-valued stochastic processes.

1.5. The droplet. We define, for a smooth function $v$ defined on $\Omega_{\delta}$ and satisfying $\partial_{n} v=0$ on $\partial \Omega_{\delta}$, the operator

$$
\mathcal{L}^{\varepsilon}(v):=\varepsilon^{2} \Delta v-f(v)+\frac{1}{\left|\Omega_{\delta}\right|} \int_{\Omega_{\delta}} f(v) d x \text { in } \Omega_{\delta},
$$

and fix the cubic nonlinearity $f$ as in the introduction.

In the next Lemma 1.5 , and for the rest of this paper $\partial_{\xi} u:=\frac{\partial}{\partial \xi} u(\cdot)$, while $\partial_{\xi}^{l} u:=\frac{\partial^{l}}{\partial \xi^{l}} u(\cdot)$ for any integer $l>1$. Note that $\dot{\partial}_{\xi} u$ is just the usual partial derivative when $u$ is considered as a function of $x$, parameterized by $\xi$ and $\varepsilon$, but when $u$ is considered as a function of local coordinates $(r, s)$, then one must take into account the fact that $r$ and $s$ both vary with $\xi$. This observation will be used in the final section where these derivatives are estimated.

Following Theorem 2.5 of 2] (p. 267), we have: 
Lemma 1.5. For any integer $K \in \mathbb{N}$ and for $\delta$ and $\varepsilon$ sufficiently small parameters satisfying

$$
\varepsilon \leq \frac{1}{2} C_{1}^{*} \delta^{2}
$$

with

$$
C_{1}^{*}:=\frac{8 \pi f^{\prime}(1)}{3 \sqrt{6}|\Omega| \int_{-1}^{1} \sqrt{F(s)} d s},
$$

there exist a droplet-like state $u=u(x, \xi, \varepsilon, K)$, a scalar (velocity) field $c=c(\xi, \varepsilon)$ and $\widetilde{\sigma}=\widetilde{\sigma}(\xi, \varepsilon)$, such that

$$
\begin{aligned}
& \mathcal{L}^{\varepsilon}(u)=\varepsilon^{2} \Delta u-f(u)+\varepsilon \widetilde{\sigma}=\varepsilon^{2} c(\xi, \varepsilon) \partial_{\xi} u+\mathcal{O}_{L^{\infty}}\left(\varepsilon^{K}\right) \text { in } \Omega_{\delta}, \\
& \partial_{n} u=0 \text { on } \partial \Omega_{\delta}, \\
& \int_{\Omega_{\delta}} u=\left|\Omega_{\delta}\right|-\pi, \\
& \widetilde{\sigma}=\varepsilon^{-1} \frac{1}{\left|\Omega_{\delta}\right|} \int_{\Omega_{\delta}} f(u) d x,
\end{aligned}
$$

where the scalar $\xi \in\left(0,\left|\partial \Omega_{\delta}\right|\right)$ is the arc-length parameter of $\partial \Omega_{\delta}$.

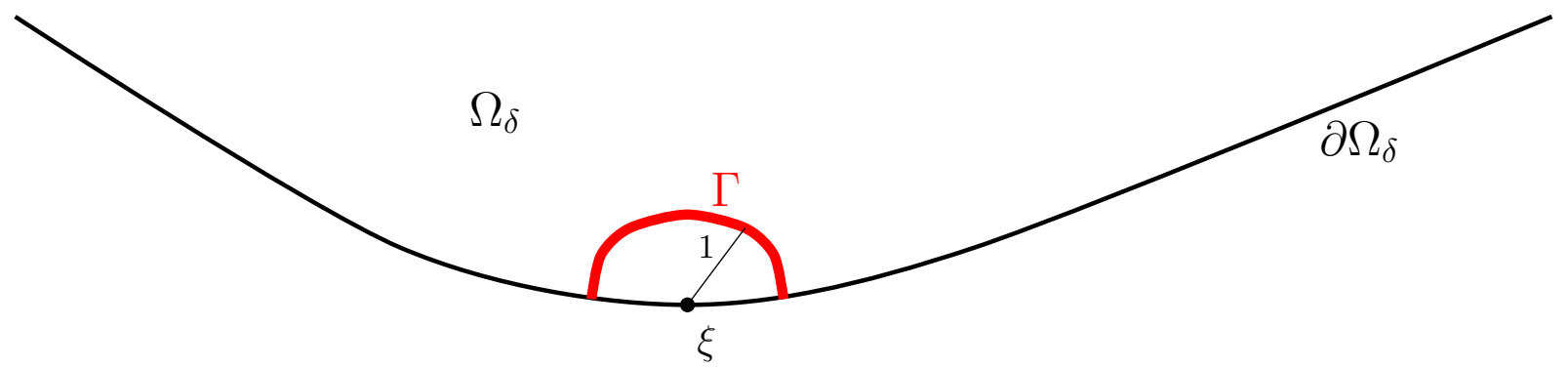

FIGURE 1.1. Droplet state in the rescaled domain $\Omega_{\delta}$ with semicircular $\operatorname{arc} \Gamma$

Idea of Proof for Lemma 1.5. The droplet is constructed, using asymptotic expansions in $\varepsilon$, as a function of $(r, s)$, with $r$ the signed distance from the interface $\Gamma$ and $s$ the arc-length along $\Gamma$, this being an approximately semicircular curve intersecting $\partial \Omega_{\delta}$ orthogonally. Additional asymptotic expansions are used near the corners where the interface meets $\partial \Omega_{\delta}$ but these are of higher order. The first order approximation to this state is $U(r / \varepsilon)$, transverse to $\Gamma$ at each point, where $U$ is the solution to

$$
\ddot{U}-f(U)=0, \quad U( \pm \infty)= \pm 1, \quad \text { such that } \int_{\mathbb{R}} R \dot{U}^{2}(R) d R=0 .
$$

Remark 1.6. In the statement of the previous lemma, we kept the notation used in Theorem 2.5 of [2] where the droplet is defined as the first component $u(x, \xi, \varepsilon)$ of the solution $(u, c, \widetilde{\sigma})$ of $(1.6)$. The Landau symbol $\mathcal{O}$ was used in [2] and there, always refers to an upper bound of the residual in the $L^{\infty}\left(\Omega_{\delta}\right)$-norm. Thus, the solution $(u, c, \widetilde{\sigma})$ of 1.6 satisfies

$$
\left\|\varepsilon^{2} \Delta u-f(u)+\varepsilon \widetilde{\sigma}(\xi, \varepsilon)-\varepsilon^{2} c(\xi, \varepsilon) \partial_{\xi} u\right\|_{L^{\infty}\left(\Omega_{\delta}\right)} \leq C \varepsilon^{K},
$$

for some constant $C>0$ independent of the sufficiently small $\varepsilon>0$, uniformly for any $\xi$ in $\left(0,\left|\partial_{\Omega_{\delta}}\right|\right)$.

Due to this property, $\varepsilon^{2} c(\xi, \varepsilon) \partial_{\xi} u$ can be used as an $\varepsilon^{K}$-approximation of $\mathcal{L}^{\varepsilon}(u)$ in the $L^{\infty}$-norm, when $u$ is the droplet state constructed in [2]. Furthermore, in view of the deterministic version of (1.4), $\varepsilon^{2} c$ gives an $\mathcal{O}\left(\varepsilon^{K}\right)$ approximation to the velocity of the droplet in the direction of $\partial_{\xi} u$, the tangent to $\partial \Omega_{\delta}$ at position $\xi$. 
Remark 1.7. The term $\widetilde{\sigma}$ of the previous lemma is denoted by $\sigma$ in [2]; we changed the notation, since the symbol $\sigma$ will be used to denote a certain variance here.

Remark 1.8. In Theorem 2.5 of [2], $\delta$ was also bounded above by a power of $\varepsilon$ but a careful reading of the proof shows that this is not needed.

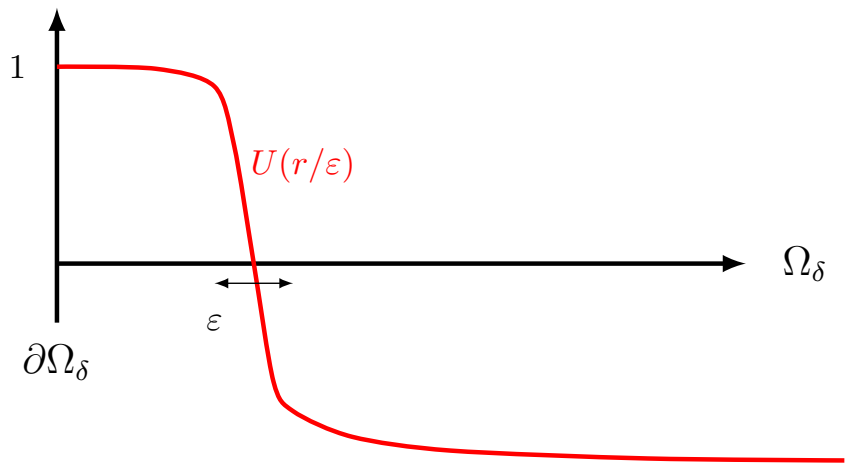

FIGURE 1.2. Sketch of a section through the droplet showing the local shape given by $U$.

According to 2] (see p. 261, and relation (2.54) on p. 262 and (4.9) on p. 297, or (2.69) on p. 267 in the initial variables) we have:

Lemma 1.9. If $c$ is the velocity from the definition of the droplet in Lemma 1.5, we have

$$
c=c(\xi, \varepsilon)=\mathcal{O}\left(\delta^{2}\right) .
$$

Moreover,

$$
c(\xi, \varepsilon)=-\frac{4}{3 \pi} g_{0} \mathcal{K}_{\Omega_{\delta}}^{\prime}(\xi) \delta[1+\mathcal{O}(\delta)]+\mathcal{O}\left(\delta^{4}\right),
$$

where $\mathcal{K}_{\Omega_{\delta}}^{\prime}(\xi)$ is the derivative of the curvature of $\partial \Omega_{\delta}$, and $g_{0}$ is a constant equal to 1 if $\varepsilon=\mathcal{O}\left(\delta^{3}\right)$. Note that $\mathcal{K}_{\Omega_{\delta}}^{\prime}(\xi)=\delta \mathcal{K}_{\Omega}^{\prime}$.

Remark 1.10. Once again we kept the notation of [2] where the Landau symbol was used.

More precisely, for sufficiently small $\delta$, there exists $C>0$ such that

$$
|c(\xi, \varepsilon)| \leq C \delta^{2},
$$

uniformly in $\varepsilon, \xi$, and

$$
c(\xi, \varepsilon)=-\frac{4}{3 \pi} g_{0} \mathcal{K}_{\Omega_{\delta}}^{\prime}(\xi) \delta\left(1+\mathcal{B}_{1}\right)+\mathcal{B}_{2},
$$

for

$$
\left|\mathcal{B}_{1}\right| \leq C \delta, \quad\left|\mathcal{B}_{2}\right| \leq C \delta^{4}
$$

uniformly in $\varepsilon, \xi$.

1.6. The manifold. We define a manifold of droplet states which are not only close to solutions, but solutions starting close to the manifold, remain close for long periods of time.

Definition 1.11. Let

$$
\mathcal{M}:=\left\{u(\cdot, \xi, \varepsilon): \xi \in\left[0,\left|\partial \Omega_{\delta}\right|\right]\right\}
$$

be the manifold consisting of the smooth functions $u$, mentioned above, i.e., functions having a droplet-like structure, satisfying (1.6). 
Obviously, $\mathcal{M}$ is a closed manifold without boundary which is diffeomorphic to the boundary $\partial \Omega_{\delta}$. Furthermore, if $\Omega_{\delta}$ is simply connected, then $\mathcal{M}$ is topologically a circle.

Remark 1.12. Our aim is to observe the dynamics of solutions to (1.4) by deriving a stochastic equation for the position of the solution's approximation (droplet); this position is identified by the parameter $\xi$ on the boundary.

We define some tubular neighborhoods of $\mathcal{M}$ in which we shall work. For $r>0$, let

$$
\begin{aligned}
& \mathcal{N}_{L^{2}}^{r}:=\left\{v \in L^{2}\left(\Omega_{\delta}\right): d_{L^{2}}(v, \mathcal{M})<r\right\}, \\
& \mathcal{N}_{H_{\varepsilon}^{1}}^{r}:=\left\{v \in H^{1}\left(\Omega_{\delta}\right): d_{H_{\varepsilon}^{1}}(v, \mathcal{M})<r\right\},
\end{aligned}
$$

where $d_{L^{2}}$ is the distance in the $L^{2}\left(\Omega_{\delta}\right)$-norm and $d_{H_{\varepsilon}^{1}}$ the distance in the norm $\|\cdot\|_{H_{\varepsilon}^{1}}: H^{1}\left(\Omega_{\delta}\right) \rightarrow \mathbb{R}^{+}$ defined by

$$
\|v\|_{H_{\varepsilon}^{1}}:=\left(\varepsilon^{2}\|\nabla v\|_{L^{2}\left(\Omega_{\delta}\right)}^{2}+\|v\|_{L^{2}\left(\Omega_{\delta}\right)}^{2}\right)^{\frac{1}{2}} .
$$

The usual Sobolev space $H^{1}\left(\Omega_{\delta}\right)$ equipped with the norm $\|\cdot\|_{H_{\varepsilon}^{1}}$ will be denoted by $H_{\varepsilon}^{1}:=\left\{H^{1}\left(\Omega_{\delta}\right),\|\cdot\|_{H_{\varepsilon}^{1}}\right\}$. For the rest of this paper $(\cdot, \cdot)$ will denote the $L^{2}\left(\Omega_{\delta}\right)$-inner product and $\|\cdot\|$ the induced $L^{2}\left(\Omega_{\delta}\right)$-norm. Moreover, $\|\cdot\|_{L^{q}}, \quad\|\cdot\|_{H^{1}}, \quad\|\cdot\|_{\infty}$ will denote the norms $\|\cdot\|_{L^{q}\left(\Omega_{\delta}\right)}, \quad\|\cdot\|_{H^{1}\left(\Omega_{\delta}\right)}, \quad\|\cdot\|_{L^{\infty}\left(\Omega_{\delta}\right)}$ respectively $\left(q \in \mathbb{N}^{*}\right)$.

Let us finally remark, that for $r$ sufficiently small (depending on $\delta$ ) there is a well defined local coordinate system in each of $\mathcal{N}_{L^{2}}^{r}$ and $\mathcal{N}_{H_{\varepsilon}^{1}}^{r}$ and the projection onto $\mathcal{M}$ is well defined and smooth.

1.7. Main Results. Our aim is the detailed study of the noise effect on the droplet's motion. We follow the main lines of the method developed in 4 for the one-dimensional stochastic Cahn-Hilliard equation but with certain differences. The problem considered is different and is posed in two dimensions, while the manifold of approximating solutions is strongly related to the geometry of domain's boundary. This consists an evidence that the method could be effective for the rigorous derivation of stochastic dynamics for various phase transition (and other) models where a manifold of approximate solutions to the deterministic equation is available.

The stochastic solution of (1.4) will be approximated by states in the manifold $\mathcal{M}$ introduced in [2] in the absence of noise. However, the problem addressed is stochastic and Itô calculus is applied in place of many of the deterministic arguments, significantly modifying the results. An important and difficult part of this manuscript is devoted to the proof of $H_{\varepsilon}^{1}$-stability for the approximate solutions. In [2], $H_{\varepsilon}^{1}$-stability was derived directly by a standard parabolic regularity estimate, while here we require more detailed stochastic estimates.

In Section 2, we analyze the dynamics of solutions $w:=w^{\varepsilon}$ of 1.4 approximated by some $u$ in $\mathcal{M}$ and written as

$$
w(t)=u(\cdot, \xi(t), \varepsilon)+v(t) .
$$

Here, $v$ is orthogonal to the manifold, i.e., $v(t) \perp_{L^{2}\left(\Omega_{\delta}\right)} \partial_{\xi}(u(\cdot, \xi(t), \varepsilon))$. Note that both $w$ and $v$ as well as $\xi$ all depend on $\varepsilon$, but, for the rest of this paper, we suppress this dependence in the notation.

We suppose that $\|v\|$ is small, and $\xi$ is a diffusion process given by

$$
d \xi=b(\xi) d t+(\sigma(\xi), d W),
$$

for some scalar field $b: \mathbb{R} \rightarrow \mathbb{R}$ and some variance $\sigma: \mathbb{R} \rightarrow H$. In Remark 2.5 we comment on the fact that it is not restrictive to assume that $\xi$ is a diffusion process.

Applying Itô calculus, we compute first $b$ and $\sigma$ exactly and then estimate their size in terms of $\varepsilon$, in order to determine the major contribution. See Theorems 2.7 2.9. and Remarks 2.11 and 2.12 .

Under the assumption of a sufficiently smooth initial condition, in Theorem 2.7 we prove that, locally in time, (i.e., up to a stopping time)

$$
d \xi \approx \varepsilon^{2} c(\xi(t), \varepsilon) d t+d \mathcal{A}_{s},
$$


where the stochastic process $\mathcal{A}_{s}$ is given as a diffusion process by the formula

$$
d \mathcal{A}_{s}=A^{-1}\left[\frac{1}{2}\left(v, \partial_{\xi}^{3}(u)\right)-\frac{3}{2}\left(\partial_{\xi}^{2} u, \partial_{\xi} u\right)\right](\mathcal{Q} \sigma, \sigma) d t+A^{-1}\left(\sigma, \mathcal{Q} \partial_{\xi}^{2} u\right) d t+A^{-1}\left(\partial_{\xi} u, d W\right)
$$

with $A:=\left\|\partial_{\xi} u\right\|^{2}-\left(v, \partial_{\xi}^{2} u\right)$. It collects all terms that arise due to the presence of noise.

Theorem 2.9 estimates the effect of noise on the local in time dynamics, that is, by the additive term $d \mathcal{A}_{s}$ which supplements the deterministic dynamics of [2] with an extra noise-induced drift and a noise term. We will see later in Remark 2.11 that for small $v$ the remaining drift terms are an Itô-Stratonovic correction, so that $\mathcal{A}_{s}$ to leading order is the Wiener process $W$ projected onto the manifold.

Since the leading order term of the deterministic dynamics is $\varepsilon^{2} c(\xi(t), \varepsilon)$ of order $\mathcal{O}\left(\varepsilon^{2}\right)$, then the noise is not always dominant, at least on time-scales of order $\varepsilon^{-2}$. Only, if the noise strength is sufficiently large, or the curvature of the boundary is locally constant, can the noise dominate (cf. Remark 2.12).

This is in contrast to the one-dimensional stochastic Cahn-Hilliard equation (see [4) where it has been proved that a noise of polynomial strength in $\varepsilon$ cannot be ignored since the deterministic dynamics are exponentially small in $\epsilon$.

In Section 3, we give sufficient conditions on the noise strength so that with high probability the solution stays close to the manifold of droplet states in both the $L^{2}$ and $H_{\varepsilon}^{1}$ norms, until time $T$ of any polynomial order of $\frac{1}{\varepsilon}$, i.e., for any $T=\varepsilon^{-q}, q \in \mathbb{N}$. This is achieved by estimating the $p$-moments of $v$ in various norms.

In our $L^{2}$-stability result of Theorem 3.5. under the assumption of $\eta_{0} \leq C \varepsilon^{2 k-2-\tilde{k}}$ for some small $\tilde{k}>0$, we prove that if $w(0)$ lies in $\mathcal{N}_{L^{2}}^{\eta \varepsilon^{k-2}}$ for some $k>5$ and some $\eta>0$ independent of $\varepsilon$, then with high probability the solution stays in a slightly larger neighborhood $\mathcal{N}_{L^{2}}^{C \varepsilon^{k-2}}(C>\eta)$ for a correspondingly long time $T=\varepsilon^{-q}$, for any arbitrarily large but fixed $q \in \mathbb{N}$.

Our problem is stochastic, so stability in the $H_{\varepsilon}^{1}$-norm is investigated analytically since we can not refer to the abstract parabolic regularity argument used in [2] in the absence of noise. More specifically, in Theorem 3.13 and its corollary, imposing the additional assumption of $\eta_{2} \leq C \varepsilon^{2 k-6}$, we show that if $w(0)$ lies in $\mathcal{N}_{L^{2}}^{\eta \varepsilon^{k-2}}$ and $\nabla w(0)$ lies in $\mathcal{N}_{L^{2}}^{\eta \varepsilon^{k-4}}$ then with high probability the solution stays in $\mathcal{N}_{H_{\varepsilon}^{1}}^{C \varepsilon^{k-3-\tilde{k}}}$ for any long time $T=\varepsilon^{-q}, q \in \mathbb{N}$, where $\tilde{k}$ is just some arbitrary small positive number. Due to this result, the local in time stochastic dynamics derived in Section 2, are proven to be valid for very long time scales. Nevertheless, we can not claim that the radius of stability is the optimal one, while we cannot improve this radius with the method of proof presented here.

The independent Section 4 involves some higher order estimates needed for the stochastic dynamics. We compute these estimates by extending the analogous lower order results of [2] which were used for the deterministic problem.

Throughout this manuscript, as many of our proofs are quite technical, we present the application of Itô calculus in full details; we hope that the interested reader may gain a wider comprehension of this stochastic technique.

Finally, let us remark, that we denote various constants all by $C$, although their value may change from line to line.

\section{StOChASTIC DYNAMiCs}

2.1. The exact stochastic equation of droplet's motion. In this section, we derive the stochastic motion on the manifold $\mathcal{M}$ following the main lines of Theorem 4.3 in [2] (p. 297), presented for the deterministic problem. For the sake of readability, we use $w$ in place of $w^{\varepsilon}$, so that problem (1.4) can be written as the following stochastic PDE

$$
d w=\mathcal{L}^{\varepsilon}(w) d t+d W .
$$

Let the position on $\partial \Omega_{\delta}$ be a diffusion process $\xi$ for the arclength given by

$$
d \xi=b(\xi) d t+(\sigma(\xi), d W),
$$


for some scalar field $b: \mathbb{R} \rightarrow \mathbb{R}$ and some variance $\sigma: \mathbb{R} \rightarrow H$ still to be determined. We will justify this ansatz later in Remark 2.5. once we obtain the specific formulae of $b$ and $\sigma$.

We approximate the solution $w$ of 1.4 by some $u=u(\cdot, \xi(t), \varepsilon)$ in $\mathcal{M}$, and write

$$
w(t)=u(\cdot, \xi(t), \varepsilon)+v(t),
$$

where $v$ is orthogonal to $\partial_{\xi} u$ in $L^{2}\left(\Omega_{\delta}\right)$. We use equation $(1.4)$ written as (2.1) to get

$$
d w=\left[\varepsilon^{2} \Delta w-f(w)+\frac{1}{\left|\Omega_{\delta}\right|} \int_{\Omega_{\delta}} f(w) d x\right] d t+d W=\left[\mathcal{L}^{\varepsilon}(u)-L v-N(u, v)\right] d t+d W,
$$

where $L$ is defined, for $v$ smooth, by

$$
L v=-\varepsilon^{2} \Delta v+f^{\prime}(u) v,
$$

so that $-L$ is the linearization of the local Allen-Cahn operator at $u$, and $N(u, v)$ collects the remaining parts, which are all the nonlinear terms and the linear terms from the non-local term that contribute only to the constant Fourier-mode. These disappear in most estimates by integrating $N$ against functions of zero average.

Differentiating the function $w=u+v$ with respect to $t$, we obtain by Itô calculus

$$
d w=\partial_{\xi} u d \xi+d v+\frac{1}{2} \partial_{\xi}^{2} u d \xi d \xi .
$$

Lemma 2.1. The Itô-correction is given by

$$
d \xi d \xi=(\mathcal{Q} \sigma(\xi), \sigma(\xi)) d t .
$$

Proof. Note that using Itô-calculus, from the definition of $\xi$ in 2.2$)$ we derive

$$
d \xi d \xi=(\sigma(\xi), d W)(\sigma(\xi), d W) .
$$

Thus, the claim follows immediately from the definition of the covariance operator. In more detail, we can use the series expansion of $W$ together with $d \beta_{i} d \beta_{j}=\delta_{i j} d t$, and derive from Parceval's identity, for arbitrary functions $p$ and $q$ the relation

$$
(p, d W)(q, d W)=\sum_{i, j} a_{i} a_{j} d \beta_{i} d \beta_{j}\left(p, e_{i}\right)\left(q, e_{j}\right)=\sum_{i} a_{i}^{2}\left(p, e_{i}\right)\left(q, e_{i}\right) d t=(\mathcal{Q} p, q) d t .
$$

Therefore, substituting 2.5 in 2.4 and by using Lemma 2.1 above, we obtain

$$
d w=\partial_{\xi} u d \xi+\frac{1}{2} \partial_{\xi}^{2} u \cdot(\mathcal{Q} \sigma, \sigma) d t+d v=\left[\mathcal{L}^{\varepsilon}(u)-L v-N(u, v)\right] d t+d W .
$$

We take the $L^{2}\left(\Omega_{\delta}\right)$-inner product of 2.8 with $\partial_{\xi} u$ and arrive at

$$
\begin{aligned}
\left\|\partial_{\xi} u\right\|^{2} d \xi+\left(d v, \partial_{\xi} u\right)= & {\left[\left(\mathcal{L}^{\varepsilon}(u), \partial_{\xi} u\right)-\left(L v, \partial_{\xi} u\right)-\left(N(u, v), \partial_{\xi} u\right)\right] d t } \\
& +\left(d W, \partial_{\xi} u\right)-\frac{1}{2}\left(\partial_{\xi}^{2} u, \partial_{\xi} u\right) \cdot(\mathcal{Q} \sigma, \sigma) d t .
\end{aligned}
$$

We differentiate in $t$ the orthogonality condition $\left(v, \partial_{\xi} u\right)=0$ and obtain by applying Itô calculus

$$
\left(d v, \partial_{\xi} u\right)+\left(v, d \partial_{\xi} u\right)+\left(d v, d \partial_{\xi} u\right)=0 .
$$

As it is demonstrated by the following lemma, by making use of the relation above we shall eliminate $\left(d v, \partial_{\xi} u\right)$ in 2.9 .

Lemma 2.2. One has

$$
\begin{aligned}
\left(d v, d\left[\partial_{\xi} u\right]\right)= & -\left(v, \partial_{\xi}^{2} u\right) d \xi-\left(\sigma, \mathcal{Q} \partial_{\xi}^{2} u\right) d t \\
& -\frac{1}{2}\left(v, \partial_{\xi}^{3} u\right) \cdot(\mathcal{Q} \sigma, \sigma) d t+\left(\partial_{\xi} u, \partial_{\xi}^{2} u\right) \cdot(\mathcal{Q} \sigma, \sigma) d t
\end{aligned}
$$


Proof. Itô calculus and relation (2.6) yield

$$
d\left[\partial_{\xi} u\right]=\partial_{\xi}^{2} u d \xi+\frac{1}{2} \partial_{\xi}^{3} u \cdot(\mathcal{Q} \sigma, \sigma) d t .
$$

Using this in (2.10), we obtain after some computations

$$
\begin{aligned}
\left(\partial_{\xi} u, d v\right) & =-\left(v, d\left[\partial_{\xi} u\right]\right)-\left(d v, d\left[\partial_{\xi} u\right]\right) \\
& =-\left(v, \partial_{\xi}^{2} u\right) d \xi-\frac{1}{2}\left(v, \partial_{\xi}^{3} u\right) \cdot(\mathcal{Q} \sigma, \sigma) d t-\left(\partial_{\xi}^{2} u, d v\right) d \xi
\end{aligned}
$$

Observing that $w=u+v$ and hence, $d v=d w-d u, 2.8$ gives

$$
\begin{aligned}
-\left(\partial_{\xi}^{2} u, d v\right) d \xi & =-\left(\partial_{\xi}^{2} u, d w\right) d \xi+\left(\partial_{\xi}^{2} u, d u\right) d \xi \\
& =-\left(\partial_{\xi}^{2} u, d W\right) d \xi+\left(\partial_{\xi}^{2} u, d u\right) d \xi \\
& =-\left(\partial_{\xi}^{2} u, d W\right) d \xi+\left(\partial_{\xi} u, \partial_{\xi}^{2} u\right) \cdot(\mathcal{Q} \sigma, \sigma) d t .
\end{aligned}
$$

Using 2.2), i.e., $d \xi=b(\xi) d t+(\sigma(\xi), d W)$, and relation 2.7) we obtain for the Itô-correction

$$
\left(\partial_{\xi}^{2} u, d W\right) d \xi=\left(\partial_{\xi}^{2} u, d W\right)(\sigma, d W)=\left(\sigma, \mathcal{Q} \partial_{\xi}^{2} u\right) d t .
$$

This yields

$$
-\left(\partial_{\xi}^{2} u, d v\right) d \xi=-\left(\sigma, \mathcal{Q} \partial_{\xi}^{2} u\right) d t+\left(\partial_{\xi} u, \partial_{\xi}^{2} u\right)(\sigma, \mathcal{Q} \sigma) d t .
$$

So, by using 2.14 in 2.12 the result follows.

Now we proceed to derive the equations of motion along the manifold. Using (2.11) in 2.9 ) we arrive at

$$
\begin{aligned}
{\left[\left\|\partial_{\xi} u\right\|^{2}-\left(v, \partial_{\xi}^{2} u\right)\right] d \xi=} & {\left[\left(\mathcal{L}^{\varepsilon}(u), \partial_{\xi} u\right)-\left(L v, \partial_{\xi} u\right)-\left(N(u, v), \partial_{\xi} u\right)\right] d t } \\
& +\left[\frac{1}{2}\left(v, \partial_{\xi}^{3} u\right)-\frac{3}{2}\left(\partial_{\xi}^{2} u, \partial_{\xi} u\right)\right](\mathcal{Q} \sigma, \sigma) d t+\left(\sigma, \mathcal{Q} \partial_{\xi}^{2} u\right) d t+\left(\partial_{\xi} u, d W\right)
\end{aligned}
$$

Let us define $A:=\left\|\partial_{\xi} u\right\|^{2}-\left(v, \partial_{\xi}^{2} u\right)$. Obviously if $A \neq 0$, then 2.15 will yield the exact dynamics.

Note that provided $v$ is sufficiently small, the nonvanishing of $A$ is obvious. We prove the following lemma which establishes this under a specific smallness assumption for $v$ and estimates the asymptotic behavior of $A^{-1}$ as $\varepsilon \rightarrow 0^{+}$.

Lemma 2.3. For $k>\frac{5}{2}$ and a fixed constant $c>0$, if $\|v\|<c \varepsilon^{k-2}$, then there exists a constant $C_{0}>0$ such that $A \geq \frac{C_{0}}{\varepsilon}$ and therefore

$$
0<A^{-1} \leq C_{0}^{-1} \varepsilon \quad \text { as } \varepsilon \rightarrow 0^{+} .
$$

Proof. From [2] (p. 297) we have the following estimates

$$
\left\|\partial_{\xi} u\right\| \geq C^{-1} \varepsilon^{-1 / 2} \quad \text { and } \quad\left\|\partial_{\xi}^{2} u\right\| \leq C \varepsilon^{-3 / 2},
$$

so,

Then, 2.17) and 2.18 yield

$$
\left\|\partial_{\xi}^{2} u\right\| \leq C \varepsilon^{-1} \varepsilon^{-1 / 2} \leq C^{3}\left\|\partial_{\xi} u\right\|^{2} \varepsilon^{-1 / 2} .
$$

$$
\begin{aligned}
A=\left\|\partial_{\xi} u\right\|^{2}-\left(v, \partial_{\xi}^{2} u\right) & \geq\left\|\partial_{\xi} u\right\|^{2}-\|v\|\left\|\partial_{\xi}^{2} u\right\| \geq\left\|\partial_{\xi} u\right\|^{2}-c \varepsilon^{k-2}\left\|\partial_{\xi}^{2} u\right\| \\
& \geq\left\|\partial_{\xi} u\right\|^{2}-c C^{3} \varepsilon^{k-2} \varepsilon^{-1 / 2}\left\|\partial_{\xi} u\right\|^{2} \\
& \geq C^{-2} \varepsilon^{-1}\left[1-c C^{3} \varepsilon^{k-5 / 2}\right] \geq C_{0} \varepsilon^{-1}
\end{aligned}
$$

for $\varepsilon$ small, provided $k>5 / 2$.

Using the previous lemma we can now prove a theorem that gives the exact stochastic o.d.e. for the droplet's motion. 
Theorem 2.4 (Exact stochastic dynamics). Let $w$ be the solution of the mass conserving Allen-Cahn equation (1.4) and let $u=u(\cdot, \xi(t), \varepsilon)$ be the solution's approximation in $\mathcal{M}$. Let $v:=w-u$ with $v$ orthogonal to $\partial_{\xi} u$ in $L^{2}\left(\Omega_{\delta}\right)$.

As long as the process $v$ is bounded by $\|v\|<c \varepsilon^{k-2}$ for some fixed $k>\frac{5}{2}$ and $c>0$, then the exact stochastic o.d.e. for the droplet's dynamics is

$$
\begin{aligned}
d \xi=A^{-1}\left[\left(\mathcal{L}^{\varepsilon}(u), \partial_{\xi} u\right)\right. & \left.-\left(L v, \partial_{\xi} u\right)-\left(N(u, v), \partial_{\xi} u\right)\right] d t \\
& +A^{-1}\left[\frac{1}{2}\left(v, \partial_{\xi}^{3} u\right)-\frac{3}{2}\left(\partial_{\xi}^{2} u, \partial_{\xi} u\right)\right](\mathcal{Q} \sigma, \sigma) d t+A^{-1}\left(\sigma, \mathcal{Q} \partial_{\xi}^{2} u\right) d t \\
& +A^{-1}\left(\partial_{\xi} u, d W\right),
\end{aligned}
$$

where

and

$$
A=\left\|\partial_{\xi} u\right\|^{2}-\left(v, \partial_{\xi}^{2} u\right)
$$

$$
\sigma=A^{-1} \partial_{\xi} u
$$

and thus, $d \xi=b d t+(\sigma, d W)$ with

$$
\begin{aligned}
b=A^{-1}\left[\left(\mathcal{L}^{\varepsilon}(u), \partial_{\xi} u\right)\right. & \left.-\left(L v, \partial_{\xi} u\right)-\left(N(u, v), \partial_{\xi} u\right)\right] \\
& +A^{-3}\left[\frac{1}{2}\left(v, \partial_{\xi}^{3} u\right)-\frac{3}{2}\left(\partial_{\xi}^{2} u, \partial_{\xi} u\right)\right]\left(\mathcal{Q} \partial_{\xi} u, \partial_{\xi} u\right)+A^{-2}\left(\partial_{\xi} u, \mathcal{Q} \partial_{\xi}^{2} u\right) .
\end{aligned}
$$

Proof. Since $v$ is sufficiently small, then the nonvanishing of $A$ follows from Lemma 2.3 . Therefore, relation 2.15 yields the exact dynamics 2.20 .

We use 2.20 and derive (2.21). Then in 2.20) we collect the ' $d t$ ' terms (drift), and replace $\sigma$ by (2.21) to obtain the formula 2.22 for $b$.

Remark 2.5. The assumption of $\xi$ being a diffusion process is not restrictive. Following the steps of the derivation backwards, it can be established that for any pair $(\xi, v)$ where $\xi$ solves (2.20) and $v$ solves

$$
d v=d\left[\partial_{\xi} u\right]+\mathcal{L}^{\varepsilon}(v+u)+f(v+u) d t+d W
$$

indeed $v \perp \partial_{\xi} u$ in $L^{2}$, and the function $w=u+v$ solves the mass conserving Allen-Cahn equation with noise. See also the analogous results for the stochastic Cahn-Hilliard equation in [4, or the detailed discussion in [37] for the stochastic Allen-Cahn equation without the mass-conservation constraint.

Remark 2.6. In the absence of noise, $W$ and $\mathcal{Q}=0$, then equation (2.20) becomes

$$
d \xi=A^{-1}\left[\left(\mathcal{L}^{\varepsilon}(u), \partial_{\xi} u\right)-\left(L v, \partial_{\xi} u\right)-\left(N(u, v), \partial_{\xi} u\right)\right] d t,
$$

and coincides with the deterministic result of [2].

Our aim now is to evaluate the contribution of the noise to dynamics; this will be achieved by estimating the additional terms in 2.20 that stem from the noise.

2.2. The approximate stochastic o.d.e. for the droplet's motion. We proceed by proving the main theorems of Section 2 that describe the droplet's exact dynamics and approximations thereof, in terms of $\varepsilon$.

Here, we need to assume bounds on the $H^{1}$-norm of $v$, in order to estimate the cubic nonlinear part of the operator; note that, considered as a Nemytski operator on $L^{2}, \tilde{f}(u)=-u^{3}$ is not continuous and $L^{2}$-smallness of $v$ does not imply that $\tilde{f}(u+v)-\tilde{f}(u)$ is even defined, let alone $o\left(\|v\|^{2}\right)$, as one would hope.

Theorem 2.7. For some $k>\frac{5}{2}$ and a fixed small $\tilde{\kappa}>0$, suppose that $w(0) \in \mathcal{N}_{L^{2}}^{\eta \varepsilon^{k-2}} \cap \mathcal{N}_{H_{\varepsilon}^{1}}^{\eta-3-\tilde{\kappa}}$ for some fixed constant $\eta$. Then, locally in time (up to the stopping time $\tau$ where $w$ leaves the neighbourhood $\mathcal{N}_{H_{\varepsilon}^{1}}^{C \varepsilon^{k-3-\tilde{\kappa}}} \cap \mathcal{N}_{L^{2}}^{C \varepsilon^{k-2}}$ for another fixed large constant $\left.C>\eta\right)$, one has

$$
d \xi=\varepsilon^{2} c(\xi(t), \varepsilon) d t+\mathcal{D}_{1} d t+d \mathcal{A}_{s}
$$


where the stochastic process $\mathcal{A}_{s}$ defined in $(1.10)$ is the part in the equation $(2.20$ for motion of $\xi$ which arises due to the presence of noise, and

$$
\mathcal{D}_{1}:=A^{-1}\left[\left(\mathcal{L}^{\varepsilon}(u), \partial_{\xi} u\right)-\left(L v, \partial_{\xi} u\right)-\left(N(u, v), \partial_{\xi} u\right)\right]-\varepsilon^{2} c(\xi(t), \varepsilon) .
$$

Furthermore,

$$
\sup _{t \in[0, \tau]}\left|\mathcal{D}_{1}\right| \leq C_{2} \varepsilon^{\mu}
$$

for $\mu:=\min \left\{2 k-\frac{11}{2}-\tilde{\kappa}, 3 k-\frac{19}{2}-2 \tilde{\kappa}, k-\frac{3}{2}\right\}$ and some constant $C_{2}>0$.

Before we prove the result, let us remark that we only assume $k>\frac{5}{2}$, which ensures the invertibility of the $A$. In that case the result of our theorem is true, but as $\mu<0$ is possible it might be useless for later results, like the $H^{1}$-stability where we then will be more restrictive and assume $k>5$. Let us just remark that (assuming $\tilde{\kappa}$ to be small) $\mu>0$ for $k>19 / 6$ and $\mathcal{D}_{1}=\mathcal{O}\left(\varepsilon^{k-3 / 2}\right)$ for $k>5$. Finally, $\mathcal{D}_{1}=\mathcal{O}\left(\varepsilon^{2}\right)$ for $k>23 / 6$.

Proof. Writing $w=u(\xi(t), \varepsilon)+v$ with $v \perp \partial u / \partial \xi$, as before and using the results of p. 297 of [2, we claim

$$
\left(\mathcal{L}^{\varepsilon}(u), \partial_{\xi} u\right)-\left(L v, \partial_{\xi}(u)\right)-\left(N(u, v), \partial_{\xi} u\right)=\left(\varepsilon^{2} c+\mathcal{D}_{2}\right)\left\|\partial_{\xi} u\right\|^{2},
$$

where we will bound

$$
\left|\mathcal{D}_{2}\right| \leq C \varepsilon^{\mu} .
$$

Indeed, observe first that by 1.6$)$ for any large $K>k$, we may choose our approximate solution family $\left\{u(x, t)=u^{\xi}(x, t ; \varepsilon, \delta)\right\}_{\xi \in\left[0,\left|\partial \Omega_{\delta}\right|\right)}$ so that

$$
\left|\left(\mathcal{L}^{\varepsilon}(u), \partial_{\xi} u\right)-c \varepsilon^{2}\left\|\partial_{\xi} u\right\|^{2}\right| \leq C \varepsilon^{K}\left\|\partial_{\xi} u\right\|_{L^{1}} .
$$

Note that 2] use the minimal $K=k$, but here, in order to simplify some of the arguments, we assume that $K$ is sufficiently large.

In addition, we have the following interpolation inequalities

$$
\|v\|_{L^{4}}^{2} \leq\|v\|_{H^{1}}\|v\|, \quad \text { and } \quad\|v\|_{L^{6}}^{3} \leq\|v\|_{H^{1}}^{2}\|v\| .
$$

Since our nonlinearity is a cubic one and $u$ is uniformly bounded and since $\int_{\Omega_{\delta}} u_{\xi} d x=\partial_{\xi} \int_{\Omega_{\delta}} u d x=0$, by the constancy of the mass over all the family, we arrive at

$$
\left|\left(N(u, v), \partial_{\xi} u\right)\right| \leq C\left(\|v\|_{H^{1}}+\|v\|_{H^{1}}^{2}\right)\|v\|\left\|\partial_{\xi} u\right\| \leq C\left(\varepsilon^{2 k-6-\tilde{\kappa}}+\varepsilon^{3 k-10-2 \tilde{\kappa}}\right) \varepsilon^{1 / 2}\left\|\partial_{\xi} u\right\|^{2},
$$

where we used

$$
\varepsilon^{-1 / 2} \leq C\left\|\partial_{\xi} u\right\|, \quad\|v\|_{H^{1}} \leq \varepsilon^{-1}\|v\|_{H_{\varepsilon}^{1}}, \quad \text { and } \quad w \in \mathcal{N}_{H_{\varepsilon}^{1}}^{C \varepsilon^{k-3-\tilde{\kappa}}} \cap \mathcal{N}_{L^{2}}^{C \varepsilon^{k-2}} .
$$

Note that this is the only part of the proof where the $H^{1}$-norm appears.

For the other term, again using $\left\|\partial_{\xi} u\right\| \geq C \varepsilon^{-1 / 2}$ and $\left\|\Delta \partial_{\xi} u\right\| \leq C \varepsilon^{-5 / 2}$ (since the bound of $\left\|u_{\xi}^{(3)}\right\|$ gives a bound of the same order in $\varepsilon$ for $\left\|\Delta \partial_{\xi} u\right\|$; see Section 4 ), we obtain

$$
\begin{aligned}
\left|\left(L v, \partial_{\xi} u\right)\right| & =\left|\left(v, L\left[\partial_{\xi} u\right]\right)\right|=\left|-\varepsilon^{2}\left(v, \Delta \partial_{\xi} u\right)+\left(v, f^{\prime}(u) \partial_{\xi} u\right)\right| \\
& \leq \varepsilon^{2}\|v\| \varepsilon^{-5 / 2}+C\|v\|\left\|\partial_{\xi} u\right\| \\
& \leq C \varepsilon^{k-5 / 2}+C \varepsilon^{k-2}\left\|\partial_{\xi} u\right\| \\
& \leq C \varepsilon^{k-3 / 2}\left\|\partial_{\xi} u\right\|^{2} .
\end{aligned}
$$

Hence, relation 2.25 follows, where $\mathcal{D}_{2}$ is estimated by (2.26).

Now it remains to relate the bound on $\mathcal{D}_{2}$ to the one on $\mathcal{D}_{1}$, which we claimed in our theorem. For short-hand notation we abbreviate

$$
B:=\left[\frac{1}{2}\left(v, \partial_{\xi}^{3} u\right)-\frac{3}{2}\left(\partial_{\xi}^{2} u, \partial_{\xi} u\right)\right]
$$


and thus, we can restate 2.15 to obtain

$$
A d \xi=\left[\left(\mathcal{L}^{\varepsilon}(u), \partial_{\xi} u\right)-\left(L v, \partial_{\xi} u\right)-\left(N(u, v), \partial_{\xi} u\right)\right] d t+B(\mathcal{Q} \sigma, \sigma) d t+\left(\sigma, \mathcal{Q} \partial_{\xi}^{2} u\right) d t+\left(\partial_{\xi} u, d W\right) .
$$

Thus, relation 2.25) yields

$$
\begin{aligned}
A d \xi= & \left(\varepsilon^{2} c(\xi, \varepsilon)+\mathcal{D}_{2}\right)\left\|\partial_{\xi} u\right\|^{2} d t \\
& +B(\mathcal{Q} \sigma, \sigma) d t+\left(\sigma, \mathcal{Q} \partial_{\xi}^{2} u\right) d t+\left(\partial_{\xi} u, d W\right) .
\end{aligned}
$$

Therefore, 1.10 gives

$$
d \xi=A^{-1}\left(\varepsilon^{2} c(\xi, \varepsilon)+\mathcal{D}_{2}\right)\left\|\partial_{\xi}(u)\right\|^{2} d t+d \mathcal{A}_{s}
$$

Hence, we get

$$
d \xi=\left(\varepsilon^{2} c(\xi, \varepsilon)+\mathcal{D}_{1}\right) d t+d \mathcal{A}_{s} .
$$

By 2.19) in the proof of Lemma 2.3. locally in time i.e. as long as $w(t) \in \mathcal{N}_{L^{2}}^{\eta \varepsilon^{k-2}}$ the following bound holds true using $k>5 / 2$

$$
A^{-1}\left\|\partial_{\xi} u\right\|^{2}=\left[1+\mathcal{O}\left(\varepsilon^{k-5 / 2}\right)\right]^{-1}=1+\mathcal{O}\left(\varepsilon^{k-5 / 2}\right) .
$$

So, from 2.29, 2.30 and 2.31), analogously to the arguments in p. 297 of [2] and since $|c(\xi, \varepsilon)| \leq C \delta^{2}$, we have from using the estimate of $\mathcal{D}_{2}$

$$
\left|A^{-1}\left(\varepsilon^{2} c+\mathcal{D}_{2}\right)\left\|\partial_{\xi} u\right\|^{2}-\varepsilon^{2} c(\xi, \varepsilon)\right| \leq C\left(\varepsilon^{k-1 / 2} \delta^{2}+\varepsilon^{\mu}\right) \leq C \varepsilon^{\mu} .
$$

Taking the supremum in 2.32 up to the stopping time $\tau$, the result follows.

Remark 2.8. Let $X$ be a random process and $k \in \mathbb{R}$. We call $X$ a process of order $\mathcal{O}\left(\varepsilon^{k}\right)$ up to a stopping time $\tau$, if

$$
\sup _{t \in[0, \tau]}|X| \leq C \varepsilon^{k},
$$

for some constant $C>0$ for all $\varepsilon>0$ sufficiently small. Then, under the assumptions of previous theorem, (2.24) can be represented as

$$
d \xi=\varepsilon^{2} c(\xi(t), \varepsilon) d t+\mathcal{O}\left(\varepsilon^{\mu}\right) d t+d \mathcal{A}_{s} .
$$

In the following theorem we shall evaluate the noise effect in the local in time stochastic dynamics (2.24) driven by the additive term $d \mathcal{A}_{s}$ defined by 1.10 which supplies the deterministic dynamics of [2] with an extra noise-induced drift and a noise term.

Theorem 2.9. Under the assumptions of Theorem 2.7. locally in time (up to the stopping time $\tau$ defined in the statement of Theorem 2.7), the noise induced terms appearing in (2.24) and given by (1.10) are estimated as follows

$$
d \mathcal{A}_{s}=\mathcal{D}_{3} d t+(\sigma, d W)
$$

with

$$
\sup _{t \in[0, \tau]}\left|\mathcal{D}_{3}\right| \leq C \eta_{1}, \quad \sup _{t \in[0, \tau]}\|\sigma\| \leq C \varepsilon^{\frac{1}{2}} .
$$

Here, $C>0$ and $\eta_{1}=\|Q\|$ is a measure of the noise strength introduced earlier.

Moreover, the dynamics equation (2.20) is represented by

$$
d \xi=\varepsilon^{2} c(\xi(t), \varepsilon) d t+A^{-2}\left(\partial_{\xi} u, \mathcal{Q} \partial_{\xi}^{2} u\right) d t+A^{-1}\left(\partial_{\xi} u, d W\right)+b_{3} d t,
$$

where

$$
\sup _{t \in[0, \tau]}\left|b_{3}\right| \leq C\left(\varepsilon^{\mu}+\eta_{1} \varepsilon^{\lambda}\right),
$$

for $\lambda:=\min \left\{1, k-\frac{5}{2}\right\}$ and $\mu$ with $\mu=k-\frac{3}{2}$ for $k>5$ defined in the previous theorem. 
Proof. We shall use some estimates proven in Section 4. For $\varepsilon$ small, by 4.1, 4.3, and 4.4 given in Theorems 4.1 and 4.2 , it holds that

$$
\begin{aligned}
& \left|\left(\partial_{\xi}^{2} u, \partial_{\xi} u\right)\right| \leq C \varepsilon^{-1}, \quad\left\|\partial_{\xi} u\right\| \leq C \varepsilon^{-\frac{1}{2}}, \\
& \left\|\partial_{\xi}^{2} u\right\| \leq C \varepsilon^{-\frac{3}{2}}, \quad\left\|\partial_{\xi}^{3} u\right\| \leq C \varepsilon^{-\frac{5}{2}}, \quad\|u\|_{L^{\infty}} \leq C .
\end{aligned}
$$

We need to estimate, by means of upper bounds in terms of $\varepsilon$, all the new terms appearing in the stochastic dynamics.

Lemma 2.3 yields

$$
\left|A^{-1}\right| \leq C \varepsilon,
$$

since locally in time we assumed that $\|v\|_{L^{2}} \leq C \varepsilon^{k-2}$.

Recall from (2.31)

$$
\left|A^{-1}\right| \cdot\left\|\partial_{\xi} u\right\|^{2} \leq C\left(1+\varepsilon^{k-5 / 2}\right) \leq C .
$$

We can estimate now the variance $\sigma$. Indeed,

$$
\|\sigma\|^{2}=\left|A^{-1}\right|^{2}\left\|\partial_{\xi} u\right\|^{2}=\left|A^{-1}\right|\left|A^{-1}\right|\left\|\partial_{\xi} u\right\|^{2} \leq C\left|A^{-1}\right| \leq C \varepsilon .
$$

Let us now turn to the bound on $\mathcal{D}_{3}$ where the terms are given by 1.10$)$. First the term $A^{-1}\left(\partial_{\xi}^{2} u, \partial_{\xi}(u)\right)(\mathcal{Q} \sigma, \sigma)$ is estimated as follows. Using the induced $L^{2}\left(\Omega_{\delta}\right)$-operator norm $\eta_{1}=\|\mathcal{Q}\|$, we get

$$
|(\mathcal{Q} \sigma, \sigma)| \leq\|\mathcal{Q}\|\|\sigma\|^{2} \leq C \eta_{1} \varepsilon .
$$

In addition, we have

$$
A^{-1}\left(\partial_{\xi}^{2} u, \partial_{\xi} u\right)(\mathcal{Q} \sigma, \sigma) \leq C \varepsilon \varepsilon^{-1}\|\mathcal{Q}\| \varepsilon=C \varepsilon \eta_{1} .
$$

Furthermore, the following estimate holds true

$$
\left|A^{-1}\left(\sigma, \mathcal{Q} \partial_{\xi}^{2} u\right)\right| \leq\left|A^{-1}\left\|A^{-1} \mid\right\| \partial_{\xi} u\|\| \mathcal{Q}\|\| \partial_{\xi}^{2} u\left\|\leq C \varepsilon^{2} \varepsilon^{-1 / 2}\right\| \mathcal{Q} \| \varepsilon^{-3 / 2}=C \eta_{1} .\right.
$$

The remaining term depending on $v$ is bounded (as long as $\|v\|_{L^{2}} \leq C \varepsilon^{k-2}$ ) by

$$
\frac{1}{2}\left|A^{-1}\left(v, \partial_{\xi}^{3}(u)\right)(\mathcal{Q} \sigma, \sigma)\right| \leq C \varepsilon\|v\| \varepsilon^{-5 / 2}\|\mathcal{Q}\|\|\sigma\|^{2}=C \varepsilon^{k-5 / 2} \eta_{1} .
$$

Hence, taking the supremum up to the stopping time $\tau$, we obtain the result for $\mathcal{D}_{3}$. The remaining bound for $d \xi$, is a combination of the bounds derived above and the bound on the drift provided by Theorem 2.7).

Remark 2.10. Obviously, the deterministic drift of $\xi=b d t+(\sigma, d W)$ is $b=\varepsilon^{2} c(\xi, \varepsilon)+\mathcal{D}_{1}+\mathcal{D}_{3}$ and thus, estimated up to a stopping time by Theorems 2.7. 2.9.

Under the assumptions of Theorem 2.9, $d \mathcal{A}_{s}$ can be represented as

$$
d \mathcal{A}_{s}=\mathcal{O}\left(\eta_{1}\right) d t+\left(\mathcal{O}_{L^{2}}\left(\varepsilon^{\frac{1}{2}}\right), d W\right)
$$

where the leading order term of the noise-induced drift $\mathcal{O}\left(\eta_{1}\right)$ of $d \mathcal{A}_{s}$ is

$$
A^{-2}\left(\partial_{\xi} u, \mathcal{Q} \partial_{\xi}^{2} u\right)+\mathcal{O}\left(\eta_{1} \varepsilon^{\lambda}\right)
$$

and the stochastic dynamics 2.20 as

$$
d \xi=\varepsilon^{2} c(\xi(t), \varepsilon) d t+A^{-2}\left(\partial_{\xi} u, \mathcal{Q} \partial_{\xi}^{2} u\right) d t+A^{-1}\left(\partial_{\xi} u, d W\right)+\mathcal{O}\left(\varepsilon^{\mu}+\eta_{1} \varepsilon^{\lambda}\right) d t .
$$

Here, using the notation of Remark 2.8, the $\mathcal{O}$-terms are stochastic processes uniformly bounded up to the stopping time $\tau$, which is here the time when $w$ leaves the neighbourhood $\mathcal{N}_{H_{\varepsilon}^{1}}^{C \varepsilon^{k-3-\kappa}} \cap \mathcal{N}_{L^{2}}^{C \varepsilon^{k-2}}$. 
2.3. Interpretation of the result. Here, we present some comments on the results derived in the section above.

Remark 2.11 (Itô-Stratonovich correction). Using the Itô-Stratonovich correction term, we obtain for a function $g(\xi)$

$$
(g, \circ d W)=\frac{1}{2}\left(g, \mathcal{Q} \partial_{\xi} g\right) d t+(g, d W)
$$

where $\mathcal{Q}$ is the covariance operator of $W$, and $\circ d W$ denotes the Stratonovic differential.

Considering Theorem 2.9. observe that $\partial_{\xi} v=\partial_{\xi} u$, since the solution $w$ is independent of $\xi$. Thus, a straightforward calculation gives

$$
d \mathcal{A}_{s}=A^{-1}\left(\partial_{\xi} u, \circ d W\right)
$$

which is the Wiener process $W$ projected onto the manifold of the small droplets.

Remark 2.12 (Boundaries of constant curvature). If the noise is small then the manifold is attractive and the motion of the droplet is approximately described up to first order by

$$
d \xi=\varepsilon^{2} c(\xi, \varepsilon) d t
$$

This is exactly the deterministic result of [2]. On timescales of order $\varepsilon^{-2}$ the droplet moves with velocity determined by the changes of the curvature of the boundary.

If the domain's boundary contains curves of constant curvature, like circular arcs or linear segments, then the main order term of the velocity field $c(\xi, \varepsilon)$, which depends on the derivative of the curvature, vanishes along these boundary parts. Hence, we expect that the droplet moves driven by a Wiener process projected to the manifold, i.e., locally this motion is like a Brownian one. Nevertheless, a rigorous proof of such a claim would demand a detailed analysis of the higher order (in $\delta$ ) corrections of $c(\xi, \varepsilon)$.

Remark 2.13 (Extremal points of curvature). Similarly to Remark 2.12, we could study the random fluctuations at the stationary points of maximal or minimal curvature. In this case, at least locally, we expect also the Brownian motion to dominate. The noise would drive the droplet away from the unstable stationary points of minimal curvature. Furthermore, the droplet would be deterministically attracted at a point of maximal curvature and noise would only induce fluctuations around it.

A result of large deviation type should hold for the droplet's exit from a point of maximal curvature. An interesting question is in which direction the droplet will exit. For small amplitude noise, we conjecture that it moves along the manifold and does not exit in the normal direction away from the boundary. Our present stability analysis does not answer this question since the case of very large time-scales (exponential), present in large deviation problems, is not covered by our results.

Remark 2.14 (Large Noise). Our approximation of $\xi$ is valid for a general noise strength $\eta_{1}=\|\mathcal{Q}\|$ as long as $\|v\|_{H_{1}^{1}} \leq C \varepsilon^{k-3-s}$ for $s>0$ arbitrarily small. If the noise strength is large, we expect the droplet to move randomly and independently from the curvature of the boundary.

But as we shall see in the sequel, we are not yet able to verify the stability of the slow manifold for relatively large noise strength. Only for a sufficiently smooth in space noise of sufficiently small strength, the attractivity result is established on arbitrarily long time intervals. As we prove, the restrictions that we must impose on the noise strength for maintaining $\|v\|_{H_{\varepsilon}^{1}} \leq C \varepsilon^{k-3-s}$, lead to a noise that does not dominate the deterministic dynamics on time-scales of order $\varepsilon^{-2}$.

More specifically, as we prove, for any $k>5$ one of the restrictions is given by

$$
\eta_{1} \leq \eta_{0} \leq C \varepsilon^{2 k-2-\sigma}, \sigma>0 \text { arbitrarily small, }
$$

resulting in a noise which is small when compared with the original dynamics of the deterministic problem, but it has a significant impact on the long time-scales we are considering.

The smallness assumption imposed on the noise stems mainly from the fact that for the moment we are not able to find an efficient way to control the nonlinearity further away from the manifold of droplet states. Moreover, the linear attractivity of this manifold is pretty weak. The complete analysis of stochastic dynamics for a much larger noise strength is a difficult open problem to be investigated in a future work. 


\section{Stochastic stability}

In this section, we establish the stability of our droplet-manifold in the $L^{2}$-norm for any polynomial times of order $\mathcal{O}\left(\varepsilon^{-q}\right), q>0$. Note that we could not apply standard large deviation type estimates, since the droplet is not exiting from a single fixed point, and we only have a manifold of approximate solutions. Moreover, results in the spirit of Berglund and Gentz [10] are not yet developed in the infinite dimensional setting.

3.1. $L^{2}$-bounds. Here, we follow the proof of 2 (p. 296) with some significant changes related to the additive noise.

We wish to show that some small tubular neighborhood of our manifold is positively invariant. Obviously, in the stochastic setting any solution will leave the neighborhood at some point. The question is, how long

does this take. Here, we are going to present a relatively simple and direct proof for the bound on the exit time.

Recall that

where $v(t) \perp_{L^{2}\left(\Omega_{\delta}\right)} \partial_{\xi} u$. Relation 2.8 gives

$$
w(t)=u(\cdot, \xi(t), \varepsilon)+v(t),
$$

$$
d w=\left[\mathcal{L}^{\varepsilon}(u)-L v-N(u, v)\right] d t+d W
$$

while

$$
d w=d u+d v=\partial_{\xi} u d \xi+\frac{1}{2} \partial_{\xi}^{2} u(Q \sigma, \sigma) d t+d v
$$

Recall the definitions

$$
\begin{aligned}
\mathcal{L}^{\varepsilon}(u) & :=\varepsilon^{2} \Delta u-f(u)+\frac{1}{\left|\Omega_{\delta}\right|} \int_{\Omega_{\delta}} f(u) d x, \quad L v:=-\varepsilon^{2} \Delta v+f^{\prime}(u) v, \\
N(u, v) & :=f(u+v)-f(u)-f^{\prime}(u) v-\frac{1}{\left|\Omega_{\delta}\right|} \int_{\Omega_{\delta}}[f(u+v)-f(u)] d x .
\end{aligned}
$$

Recall from Lemma 1.5 that (for any large $K>k$ )

$$
\mathcal{L}^{\varepsilon}(u)=c(\xi, \varepsilon) \varepsilon^{2} \partial_{\xi} u+\mathcal{B}, \quad \text { with }\|\mathcal{B}\|_{L^{\infty}} \leq C\left|\varepsilon^{K}\right| .
$$

Solving (3.1) and (3.2) for $d v$ and substituting (3.3), we obtain the dynamics of $v$ given by the next lemma.

Lemma 3.1. Consider a solution $w(t)=u(\cdot, \xi(t), \varepsilon)+v(t)$ with $v(t) \perp \partial_{\xi} u$ and $\xi$ being our diffusion process on the manifold, then

$$
d v=\left[c \varepsilon^{2} \partial_{\xi} u+\mathcal{B}-L v-N(u, v)\right] d t-\partial_{\xi} u d \xi-\frac{1}{2} \partial_{\xi}^{2} u(\mathcal{Q} \sigma, \sigma) d t+d W .
$$

Let us now turn to the estimate of the $L^{2}$-norm $\|v\|^{2}$. First, since $\left(\partial_{\xi} u, v\right)=0$, we obtain

$$
(d v, v)=[(\mathcal{B}, v)-(L v, v)-(N(u, v), v)] d t-\frac{1}{2}\left(\partial_{\xi}^{2}(u), v\right)(\mathcal{Q} \sigma, \sigma) d t+(v, d W) .
$$

In view of 3.5 we first observe that Itô calculus gives

$$
d\|v\|^{2}=d(v, v)=2(v, d v)+(d v, d v) .
$$

Since $d \xi=b d t+(\sigma, d W)$ and using the Itô-correction $(d W, d W)=\operatorname{trace}(\mathcal{Q}) d t=\eta_{0} d t$, by Itô-calculus we derive

$$
\begin{aligned}
(d v, d v) & =(d W, d W)-2\left(d W, \partial_{\xi} u\right) d \xi+\left(\partial_{\xi} u, \partial_{\xi} u\right) d \xi d \xi \\
& =\eta_{0} d t-2\left(\partial_{\xi}(u), \mathcal{Q} \sigma\right) d t+\left\|\partial_{\xi} u\right\|^{2}(\sigma, \mathcal{Q} \sigma) d t \\
& \leq C \eta_{0} d t
\end{aligned}
$$

where we used in the last step that $\left\|\partial_{\xi} u\right\| \leq C \varepsilon^{-1 / 2},\|\mathcal{Q}\|=\eta_{1} \leq \eta_{0}$ and $\|\sigma\| \leq C \varepsilon^{1 / 2}$. 
Finally, we have

$$
\frac{1}{2} d\|v\|^{2} \leq(v, d v)+C \eta_{0} d t .
$$

Note that the inequality above for the stochastic differential only makes sense in an integral form. Moreover, any inequality presented in the following only involves bounds on the drift of the stochastic differentials, but not on the diffusion.

In order to proceed, we now bound the drift terms in (3.5). Obviously, we have

$$
(\mathcal{B}, v) d t \leq C \varepsilon^{K}\|v\| d t .
$$

Furthermore, for the quadratic form of the linear operator the following lemma based on [2] holds true.

Lemma 3.2. There exists some $\nu_{0}>0$ such that for all $v \perp \partial_{\xi} u$

$$
-(L v, v) \leq-\nu_{0} \varepsilon^{2}\|v\|_{H_{\varepsilon}^{1}}^{2} .
$$

Let us remark that any improvement in the spectral gap immediately yields an improvement in the noise-strength that we can effectively study.

Proof. From the main spectral theorem of [2], we have, for some constant $\tilde{\nu}>0$,

$$
-(L v, v) \leq-\tilde{\nu} \varepsilon^{2}\|v\|^{2} .
$$

Using that $u$ is bounded together with the spectral theorem, we find that for any $\gamma>0$ and $\varepsilon$ sufficiently small

$$
\begin{aligned}
-(L v, v) & =-\gamma \varepsilon^{2}(L v, v)-\left(1-\gamma \varepsilon^{2}\right)(L v, v) \\
& \leq-\gamma \varepsilon^{4}\|\nabla v\|^{2}+\gamma \varepsilon^{2}\left\|f^{\prime}(u)\right\|_{\infty}\|v\|^{2}-\left(1-\gamma \varepsilon^{2}\right) \tilde{\nu} \varepsilon^{2}\|v\|^{2} \\
& \leq-\gamma \varepsilon^{4}\|\nabla v\|^{2}-\varepsilon^{2}\left(1-\gamma \varepsilon^{2}\right) \tilde{\nu}\|v\|^{2}+C \varepsilon^{2} \gamma\|v\|^{2} \\
& =-\gamma \varepsilon^{2}\|v\|_{H_{\varepsilon}^{1}}^{2}+\varepsilon^{2}\|v\|^{2}\left[\gamma-\left(1-\gamma \varepsilon^{2}\right) \tilde{\nu}+C \gamma\right] \\
& \leq-\varepsilon^{2} \gamma\|v\|_{H_{\varepsilon}^{1}}^{2}
\end{aligned}
$$

provided we choose $\gamma$ sufficiently small, which then defines $\nu_{0}$. Here we need to fix $0<\gamma<\tilde{\nu} /\left(1+C+\varepsilon^{2} \tilde{\nu}\right)$ to obtain that

$$
\left[\gamma-\left(1-\gamma \varepsilon^{2}\right) \tilde{\nu}+c \gamma\right]<0
$$

Finally, we consider the term $-(N(u, v), v) d t$. First, the non-local contribution on the constant mode vanishes, as $\int_{\Omega_{\delta}} v d x=0$. Moreover, since $u$ is uniformly bounded, we obtain

$$
(N(u, v), v)=\int_{\Omega_{\delta}}\left(3 u v^{3}+v^{4}\right) d x \geq-C \int_{\Omega_{\delta}}|v|^{3} d x+\int_{\Omega_{\delta}} v^{4} d x .
$$

Hence, we arrive at

$$
-(N(u, v), v) d t \leq\left(C \int_{\Omega_{\delta}}|v|^{3} d x-\int_{\Omega_{\delta}} v^{4} d x\right) d t
$$

Also we have

$$
\left(\partial_{\xi}^{2} u, v\right)(\mathcal{Q} \sigma, \sigma) d t \leq C \varepsilon^{-3 / 2}\|v\| \eta_{1} \varepsilon^{1 / 2} \varepsilon^{1 / 2} d t=C \varepsilon^{-1 / 2} \eta_{1}\|v\| d t .
$$

Using now the relations (3.6), (3.7), (3.8), (3.9) and (3.10) in (3.5) we get

$$
d\|v\|^{2} \leq\left[C \eta_{0}+C \varepsilon^{K}\|v\|-2 \nu_{0} \varepsilon^{2}\|v\|_{H_{\varepsilon}^{1}}^{2}+C \int_{\Omega_{\delta}}|v|^{3} d x-2 \int_{\Omega_{\delta}} v^{4} d x+C \varepsilon^{-1 / 2} \eta_{1}\|v\|\right] d t+2(v, d W) .
$$


By the Gagliardo-Nirenberg inequality and since $\varepsilon \ll 1$, it follows that

$$
\begin{aligned}
C \int_{\Omega_{\delta}}|v|^{3} d x & =C\|v\|_{L^{3}}^{3} \leq \tilde{C}\|v\|_{H_{1}}\|v\|^{2} \\
& \leq C_{2} \frac{1}{\varepsilon}\|v\|_{H_{\varepsilon}^{1}}\|v\|^{2} \leq\|v\|_{H_{\varepsilon}^{1}}\|v\| \nu_{0} \varepsilon^{2} \leq \nu_{0} \varepsilon^{2}\|v\|_{H_{\varepsilon}^{1}}^{2},
\end{aligned}
$$

provided that for $\hat{C} \geq C_{2}$

$$
\|v\| \leq \frac{\nu_{0} \varepsilon^{3}}{\hat{C}} .
$$

Note that this is the point where the condition $k>5$ will finally appear, since if $\|v\| \leq C \varepsilon^{k-2}$ for $k>5$ then indeed $\|v\| \leq \frac{\nu_{0} \varepsilon^{3}}{\hat{C}}$.

Using (3.12) in 3.11) and the fact that $-\|v\|_{H_{\varepsilon}^{1}}^{2} \leq-\|v\|^{2}$ we arrive at

$$
\begin{aligned}
d\|v\|^{2} & \leq\left[C \eta_{0}+C \varepsilon^{K}\|v\|-\nu_{0} \varepsilon^{2}\|v\|_{H_{\varepsilon}^{1}}^{2}+C \varepsilon^{-1 / 2} \eta_{1}\|v\|\right] d t+2(v, d W) \\
& \leq\left[C \eta_{0}+\frac{C^{2}}{2 \nu_{0} \varepsilon^{2}}\left(\varepsilon^{K}+\varepsilon^{-1 / 2} \eta_{1}\right)^{2}-\frac{\nu_{0} \varepsilon^{2}}{2}\|v\|^{2}\right] d t+2(v, d W) .
\end{aligned}
$$

So, recalling that we only bounded the drift part of the stochastic differentials, we obtain the following lemma.

Lemma 3.3. Up to a stopping time, where $\|v\| \leq \frac{\nu_{0} \varepsilon^{3}}{\hat{C}}$, it holds that

$$
d\|v\|^{2}=\mathcal{D}_{4} d t+2(d W, v),
$$

with

$$
\mathcal{D}_{4} \leq C_{\varepsilon}-\frac{\nu_{0} \varepsilon^{2}}{2}\|v\|^{2} \quad \text { where } \quad C_{\varepsilon}:=C \eta_{0}+\frac{C^{2}}{2 \nu_{0} \varepsilon^{2}}\left(\varepsilon^{K}+\varepsilon^{-\frac{1}{2}} \eta_{1}\right)^{2} .
$$

Remark 3.4. Here, $\hat{C}$ and $C$ are the specific constants appearing in the proof of lemma.

3.2. Long-time $L^{2}$-stability. Let us define the stopping time $\tau^{\star}$ as the exit-time from a $L^{2}$-neighborhood of radius $B$ of the manifold before time $T$

$$
\tau^{\star}:=\inf \{t \in[0, T]:\|v(s)\| \leq B \text { for } 0 \leq s<t\}
$$

with the convention that

$$
\tau^{\star}=T, \quad \text { if } \quad\|v(t)\| \leq B \text { for all } t \in[0, T],
$$

and thus, the solution has not exit before $T$.

Recall that by Lemma $3.3 v$ satisfies an inequality of the form

$$
d\|v\|^{2} \leq\left[C_{\varepsilon}-a\|v\|^{2}\right] d t+2(v, d W),
$$

for all $t \leq \tau^{\star}$, provided that $B \leq \frac{\nu_{0} \varepsilon^{3}}{\hat{C}}$. Thus from now on, we fix

$$
B=C \varepsilon^{k-2} \quad \text { for } k>5 .
$$

More specifically, from 3.14

$$
a:=\frac{\nu_{0} \varepsilon^{2}}{2} \quad \text { and } \quad C_{\varepsilon}:=C \eta_{0}+\frac{C_{0}^{2}}{2 \nu_{0} \varepsilon^{2}}\left(\varepsilon^{K}+\varepsilon^{-1 / 2} \eta_{1}\right)^{2} .
$$

So, for all $t \leq \tau^{\star}$,

$$
\|v(t)\|^{2}+a \int_{0}^{t}\|v(s)\|^{2} d s \leq\|v(0)\|^{2}+C_{\varepsilon} t+2 \int_{0}^{t}(v, d W) .
$$


Using the fact that stopped stochastic integrals for deterministically bounded stopping times still have mean value 0 (referring to optimal stopping of martingales), we obtain

$$
\mathbb{E}\left\|v\left(\tau^{\star}\right)\right\|^{2}+a \mathbb{E} \int_{0}^{\tau^{\star}}\|v(s)\|^{2} d s \leq\|v(0)\|^{2}+C_{\varepsilon} T,
$$

where we used that $\tau^{\star} \leq T$ by definition.

We can extend (3.15) above to higher powers by applying Itô's formula to higher powers of $\|v\|^{2}$, where we calculate the Itô correction $\left[d\|v\|^{2}\right]^{2}=(v, \mathcal{Q} v)$ using Lemma 3.3 . We obtain

$$
\begin{aligned}
d\|v\|^{2 p} & =p\|v\|^{2 p-2} d\|v\|^{2}+p(p-1)\|v\|^{2 p-4} d\|v\|^{2} d\|v\|^{2} \\
& =p\|v\|^{2 p-2} d\|v\|^{2}+4 p(p-1)\|v\|^{2 p-4}(v, \mathcal{Q} v) d t \\
& \leq p\|v\|^{2 p-2}\left[C_{\varepsilon}-a\|v\|^{2}\right] d t+4 p(p-1)\|v\|^{2 p-2}\|\mathcal{Q}\| d t+2 p\|v\|^{2 p-2}(v, d W) .
\end{aligned}
$$

Hence, for all integers $p>1$, we arrive at

$$
\|v(t)\|^{2 p}+p a \int_{0}^{t}\|v(s)\|^{2 p} d s \leq\|v(0)\|^{2 p}+C\left(C_{\varepsilon}+\|\mathcal{Q}\|\right) \int_{0}^{t}\|v\|^{2 p-2} d t+2 p \int_{0}^{t}\|v\|^{2 p-2}(v, d W),
$$

provided $t \leq \tau^{\star}$. Here and in the sequel, we denote all constants depending explicitly on $p$ only by $C$.

Now again by evaluation at the stopping time and taking expectation, we obtain

$$
\mathbb{E}\left\|v\left(\tau^{\star}\right)\right\|^{2 p} \leq\|v(0)\|^{2 p}+C\left(C_{\varepsilon}+\|\mathcal{Q}\|\right) \cdot \mathbb{E} \int_{0}^{\tau^{\star}}\|v\|^{2 p-2} d t,
$$

and

$$
a \mathbb{E} \int_{0}^{\tau^{\star}}\|v(s)\|^{2 p} d s \leq \frac{1}{p}\|v(0)\|^{2 p}+C\left(C_{\varepsilon}+\|\mathcal{Q}\|\right) \cdot \mathbb{E} \int_{0}^{\tau^{\star}}\|v\|^{2 p-2} d t .
$$

Let us now assume that the following quantities are bounded and small

$$
q=\frac{C_{\varepsilon}+\|\mathcal{Q}\|}{a} \ll 1 \quad \text { and } \quad\|v(0)\|^{2} \leq q \ll B^{2} .
$$

An induction argument yields

$$
\begin{aligned}
\frac{1}{p} \mathbb{E}\left\|v\left(\tau^{\star}\right)\right\|^{2 p} & \stackrel{3.16}{\leq} \frac{1}{p}\|v(0)\|^{2 p}+C\left(C_{\varepsilon}+\|\mathcal{Q}\|\right) \cdot \mathbb{E} \int_{0}^{\tau^{\star}}\|v\|^{2 p-2} d t \\
& =\frac{1}{p}\|v(0)\|^{2 p}+C \cdot q a \cdot \mathbb{E} \int_{0}^{\tau^{\star}}\|v\|^{2 p-2} d t \\
& \stackrel{3.17}{\leq} \frac{1}{p}\|v(0)\|^{2 p}+C q\|v(0)\|^{2 p-2}+C q^{2} a \cdot \mathbb{E} \int_{0}^{\tau^{\star}}\|v\|^{2 p-4} d t \\
& \leq C q\|v(0)\|^{2 p-2}+C q^{2} a \cdot \mathbb{E} \int_{0}^{\tau^{\star}}\|v\|^{2 p-4} d t \\
& \leq \ldots \\
& \leq C q^{p-2}\|v(0)\|^{4}+C q^{p-1} a \cdot \mathbb{E} \int_{0}^{\tau^{\star}}\|v\|^{2} d t \\
& \leq C q^{p-2}\|v(0)\|^{4}+C q^{p-1}\left[\|v(0)\|^{2}+C_{\varepsilon} T\right] \\
& \leq C q^{p}+C a q^{p} T, \quad \text { since } C_{\varepsilon} \leq a q .
\end{aligned}
$$

Using Chebychev's inequality, we finally arrive at

$$
\begin{aligned}
\mathbb{P}\left(\tau^{\star}<T\right) & =\mathbb{P}\left(\left\|v\left(\tau^{\star}\right)\right\| \geq B\right) \leq B^{-2 p} \mathbb{E}\left\|v\left(\tau^{\star}\right)\right\|^{2 p} \\
& \leq C B^{-2 p}\left[q^{p}+a q^{p} T\right]=C\left(\frac{q}{B^{2}}\right)^{p}+C a\left(\frac{q}{B^{2}}\right)^{p} T .
\end{aligned}
$$


Therefore, we obtain the following $L^{2}$-stability theorem.

Theorem 3.5. Consider the exit time

$$
\tau^{\star}:=\inf \left\{t \in\left[0, T_{\varepsilon}\right]:\|v(t)\|>C \varepsilon^{k-2}\right\},
$$

with $T_{\varepsilon}:=\varepsilon^{-N}$ for arbitrary fixed large $N>0$. Fix

$$
k>5 \text { and }\|v(0)\|<\eta \varepsilon^{k-2} .
$$

Also, assume that the noise satisfies

$$
\eta_{0} \leq C \varepsilon^{2 k-2+\tilde{k}}
$$

for some fixed $\tilde{k}>0$ arbitrarily small. Then the probability $\mathbb{P}\left(\tau^{\star}<T_{\varepsilon}\right)$ is smaller than any power of $\varepsilon$, as $\varepsilon \rightarrow 0^{+}$. Thus, for very large times the solution stays close to the manifold with high probability.

Proof. The claim follows from inequality $(3.18)$ if $q / B^{2} \leq \varepsilon^{\tilde{\kappa}}$.

Indeed, using the definitions of $C_{\varepsilon}, a=C \varepsilon^{2}$ and $B=C \varepsilon^{k-2}$, and since $K>k$, we have

$$
q=\frac{C_{\varepsilon}+\|\mathcal{Q}\|}{a} \leq C\left[\eta_{0} \varepsilon^{-2}+\varepsilon^{-4}\left(\varepsilon^{2 K}+\varepsilon^{-1} \eta_{1}^{2}\right)\right]
$$

So, we get

$$
q / B^{2}=\eta_{0} \varepsilon^{2-2 k}+\varepsilon^{2(K-k)}+\varepsilon^{-1-2 k} \eta_{1}^{2} \leq C \varepsilon^{\tilde{\kappa}},
$$

since $\eta_{1} \leq \eta_{0}$ and $k>5$.

Remark 3.6. The stability result presented so far does not state that the local in time stochastic dynamics for $\xi$ given by Theorem 2.7 and Theorem 2.9 hold with high probability for a long time. For this we need to prove stochastic stability in the $H_{\varepsilon}^{1}$-norm. As we rely for simplicity of presentation on the direct application of Itô's formula, this will only be achieved for a noise $\dot{W}$ sufficiently regular in space.

Remark 3.7. The presence of the very small $\tilde{\kappa}$ in the conditions of $\eta_{0}$ is only for simplicity. Instead of having the $\tilde{\kappa}$ here, we could use $B:=\varepsilon^{2 k-4-\tilde{\kappa}}$. This would yield the same result but for a slightly smaller neighborhood for $v$.

We need the gap created by $\tilde{\kappa}$ in order to control the probability and to obtain very large time-scales in the stability result. This is the reason why the case $k=5$, included in the deterministic result of [2], is not covered by our analysis and we can thus, only consider $k>5$.

3.3. Estimates in the $H_{\varepsilon}^{1}$-norm. As we can not rely on bounds of the linearized operator in $H_{\varepsilon}^{1}$-norm, we shall use instead the previously established $L^{2}$-stability result given by Theorem 3.5 . Nevertheless, in order to bound the $H_{\varepsilon}^{1}$-norm of the stochastic solution over a very long time-scale, we can allow the use of a larger tube bounding $\nabla v$. But, as we shall see in the sequel, this will further limit the noise we can consider since we obtain additional restrictions for the size of $\eta_{2}$.

Let $w$ be the solution of the mass conserving stochastic Allen-Cahn equation (1.4), then we defined

$$
w=u+v, \quad u \in \mathcal{M}, \quad v \perp_{L^{2}\left(\Omega_{\delta}\right)} \partial_{\xi} u .
$$

Moreover, recall (3.4)

with

$$
d v=\left[c \varepsilon^{2} \partial_{\xi} u+\mathcal{B}-L v-N(u, v)\right] d t-\partial_{\xi} u d \xi-\frac{1}{2} \partial_{\xi}^{2} u(\mathcal{Q} \sigma, \sigma) d t+d W,
$$

$$
L v+N(u, v)=-\varepsilon^{2} \Delta v+f(u+v)-f(u)-\frac{1}{\left|\Omega_{\delta}\right|} \int_{\Omega_{\delta}}[f(u+v)-f(u)] d x .
$$

We consider first the following relation by Itô-formula

$$
\begin{aligned}
d\|\nabla v\|^{2} & =2(\nabla v, d \nabla v)+(\nabla d v, \nabla d v) \\
& =-2(\Delta v, d v)+(\nabla d v, \nabla d v),
\end{aligned}
$$

where we used integration by parts and the Neumann boundary condition of $v$. 
Observe that by series expansion of the Wiener process $W$ and since

$$
\left\|e_{k}\right\|=1, \quad\|\sigma\| \leq C \varepsilon^{1 / 2}, \quad\left\|\nabla \partial_{\xi} u\right\| \leq C \varepsilon^{-3 / 2},
$$

we obtain the following bound on an Itô-correction term

$$
\begin{aligned}
\left(\nabla \partial_{\xi} u, \nabla d W\right)(\sigma, d W) & =\sum_{k} \alpha_{k}^{2}\left(\nabla \partial_{\xi} u, \nabla e_{k}\right)\left(\sigma, e_{k}\right) d t \\
& \leq \sum_{k} \alpha_{k}\left\|\nabla e_{k}\right\| \alpha_{k}\left\|\nabla \partial_{\xi} u\right\|\|\sigma\| d t \\
& \leq \eta_{2}^{1 / 2} \eta_{0}^{1 / 2}\left\|\nabla \partial_{\xi} u\right\|\|\sigma\| d t \\
& \leq C\left(\varepsilon^{-2} \eta_{0}+\eta_{2}\right) d t .
\end{aligned}
$$

Thus, since $\eta_{1} \leq \eta_{0}$ we derive the following bound for the Itô-correction term of 3.19

$$
\begin{aligned}
(\nabla d v, \nabla d v) & =\left\|\nabla \partial_{\xi} u\right\|^{2}(d \xi)^{2}-2\left(\nabla \partial_{\xi} u, \nabla d W\right) d \xi+(\nabla d W, \nabla d W) \\
& =\left\|\nabla \partial_{\xi} u\right\|^{2}(\mathcal{Q} \sigma, \sigma) d t-2\left(\nabla \partial_{\xi} u, \nabla d W\right)(\sigma, d W)+\operatorname{trace}(\Delta \mathcal{Q}) \\
& \leq C\left(\varepsilon^{-2} \eta_{1}+\eta_{2}+\varepsilon^{-2} \eta_{0}+\eta_{2}\right) d t \\
& \leq C\left(\varepsilon^{-2} \eta_{0}+\eta_{2}\right) d t .
\end{aligned}
$$

Considering the other mixed term in $(3.19)$ and using that $(1, \Delta v)=0$, we obtain the following relation

$$
\begin{aligned}
-(\Delta v, d v)= & -\left(\Delta v, \mathcal{B}+\varepsilon^{2} \Delta v\right) d t-(\Delta v,-f(u+v)+f(u)) d t \\
& +\left(\Delta v, \partial_{\xi} u \cdot\left[b-c \varepsilon^{2}\right]+\frac{1}{2} \partial_{\xi}^{2} u \cdot(\mathcal{Q} \sigma, \sigma)\right) d t-\left(\Delta v,-\partial_{\xi} u \cdot(\sigma, d W)+d W\right) \\
= & T_{1}+T_{2}+T_{3}+T_{4} .
\end{aligned}
$$

Now we need to bound all four terms $T_{i}$ separately. First, we estimate the diffusion in the martingale term $T_{4}$. Note that below we only integrate by parts once, as we only know that $v$ satisfies Neumann boundary conditions. We get for $\mathcal{A}:=-\left(\nabla v, \nabla \partial_{\xi} u\right) \sigma$

$$
\begin{aligned}
T_{4} & =\left(\Delta v, \partial_{\xi} u\right)(\sigma, d W)-(\Delta v, d W)=-\left(\nabla v, \nabla \partial_{\xi} u\right)(\sigma, d W)+(\nabla v, \nabla d W) \\
& =\left(-\left(\nabla v, \nabla \partial_{\xi} u\right) \sigma, d W\right)+(\nabla v, \nabla d W) \\
& =(\mathcal{A}, d W)+(\nabla v, d \nabla W),
\end{aligned}
$$

where

$$
|\mathcal{A}|=\left|\left(\nabla v, \nabla \partial_{\xi} u\right) \sigma\right| \leq C \varepsilon^{-1}\|\nabla v\| .
$$

For $T_{1}$ and for $K>0$ sufficiently large, we obtain by Lemma 1.5 that

$$
\begin{aligned}
T_{1} & =-\left(\Delta v,\left[\mathcal{B}+\varepsilon^{2} \Delta v\right]\right) d t=-(\Delta v, \mathcal{B}) d t-\varepsilon^{2}\|\Delta v\|^{2} d t \\
& \leq C \varepsilon^{K}\|\Delta v\| d t-\varepsilon^{2}\|\Delta v\|^{2} d t .
\end{aligned}
$$

For $T_{3}$ we have

$$
\begin{aligned}
T_{3} & =\left(\Delta v, \partial_{\xi} u\left[b-c \varepsilon^{2}\right]+\frac{1}{2} \partial_{\xi}^{2} u(\mathcal{Q} \sigma, \sigma)\right) d t=\left(\Delta v, \partial_{\xi} u\right)\left[b-c \varepsilon^{2}\right] d t+\frac{1}{2}\left(\partial_{\xi}^{2} u, \Delta v\right)(\mathcal{Q} \sigma, \sigma) d t \\
& \leq C\|\Delta v\| \varepsilon^{-1 / 2}\left[b-c \varepsilon^{2}\right] d t+C \varepsilon^{-1 / 2} \eta_{0}\|\Delta v\| d t .
\end{aligned}
$$

Here, note that we can write $b=\varepsilon^{2} c(\xi, \varepsilon)+\mathcal{D}_{1}+\mathcal{D}_{3}$ with bounds on the $\mathcal{D}_{i}$ given by Theorems 2.7, 2.9 up to a stopping time. 
For $T_{2}$ we derive

$$
\begin{aligned}
T_{2} & =-(\Delta v,-f(u+v)+f(u)) d t=\left(\Delta v,-v+3 u^{2} v+3 u v^{2}+v^{3}\right) d t \\
& =\left(\|\nabla v\|^{2}-\int \nabla\left(3 u^{2} v+3 u v^{2}+v^{3}\right) \cdot \nabla v d x\right) d t \\
& =\left(\|\nabla v\|^{2}-\int\left(3 u^{2}+6 u v+3 v^{2}\right)|\nabla v|^{2} d x-\int\left(6 u v+3 v^{2}\right)(\nabla u \cdot \nabla v) d x\right) d t \\
& \leq\left(\|\nabla v\|^{2}-\int\left(6 u v+3 v^{2}\right)(\nabla u \cdot \nabla v) d x\right) d t .
\end{aligned}
$$

We observe, cf. Remark 4.3 that $\|\nabla u\|_{\infty} \leq C \varepsilon^{-1}$ (since $\left\|\partial_{\xi} u\right\|_{\infty} \leq C \varepsilon^{-1}$, cf. [2]). In addition, $u$ is uniformly bounded in $\varepsilon$. Furthermore, the Gagliardo-Nirenberg inequality gives

$$
\left\|v^{2}\right\|=\|v\|_{4}^{2} \leq\left(C\|v\|_{H_{1}}^{1 / 2}\|v\|^{1 / 2}\right)^{2}=C\|v\|_{H_{1}}\|v\| \leq C\|v\|^{2}+C\|\nabla v\|\|v\|
$$

while the following interpolation inequality holds true

$$
\|\nabla v\|^{2}=-(\Delta v, v) \leq\|\Delta v\|\|v\| .
$$

Using the previous estimates we arrive at

$$
\begin{aligned}
T_{2} & \leq\left(\|\nabla v\|^{2}+3\left\|v^{2}\right\|\|\nabla u\|_{\infty}\|\nabla v\|+6\|v\|\|u\|_{\infty}\|\nabla u\|_{\infty}\|\nabla v\|\right) d t \\
& \leq\left(\|\nabla v\|^{2}+C \varepsilon^{-1}\left\|v^{2}\right\|\|\nabla v\|+C \varepsilon^{-1}\|v\|\|\nabla v\|\right) d t \\
& \leq\left(\|\nabla v\|^{2}+C \varepsilon^{-1}\|v\|\|\nabla v\|^{2}+C \varepsilon^{-1}\|v\|^{2}\|\nabla v\|+C \varepsilon^{-1}\|v\|\|\nabla v\|\right) d t .
\end{aligned}
$$

So, by relation 3.20 , we derive

$$
\begin{aligned}
d\|\nabla v\|^{2} \leq & C \varepsilon^{K}\|\Delta v\| d t-2 \varepsilon^{2}\|\Delta v\|^{2} d t+2 T_{2} \\
& +C\|\Delta v\| \varepsilon^{-1 / 2}\left|b-c \varepsilon^{2}\right| d t+C \varepsilon^{-1 / 2} \eta_{0}\|\Delta v\| d t+C \varepsilon^{-2} \eta_{0}+\eta_{2} d t \\
& +(2 \mathcal{A}, d W)+2(\nabla v, \nabla d W),
\end{aligned}
$$

for $T_{2}$ estimated by 3.22 and $\mathcal{A}$ by (3.21). Therefore, Young's inequality yields

$$
\begin{aligned}
d\|\nabla v\|^{2} \leq & -\varepsilon^{2}\|\Delta v\|^{2} d t+2 T_{2} \\
& +C\left(\varepsilon^{2 K-2}+\varepsilon^{-3}\left|b-c \varepsilon^{2}\right|^{2}+\varepsilon^{-3} \eta_{0}^{2}+\varepsilon^{-2} \eta_{0}+\eta_{2}\right) d t \\
& +2(\mathcal{A}, d W)+2(\nabla v, \nabla d W) .
\end{aligned}
$$

In order to proceed using the estimate of $T_{2}$ given by 3.22 where the $L^{2}$-norm of $v$ is also involved, we shall use the $L^{2}$-stability result proven so far, observing evolution in time as long as $\|v\|$ is not too large.

Definition 3.8. Let $k>5$ and $\tilde{\kappa}>0$ small. For some given large $T_{\varepsilon}$, we define the stopping time

$$
\tau_{\varepsilon}=\inf \left\{t \in\left[0, T_{\varepsilon}\right]:\|\nabla v(t)\|>C_{0} \varepsilon^{k-4-\tilde{\kappa}} \text { or }\|v(t)\|>C_{0} \varepsilon^{k-2}\right\} .
$$

Here, $C_{0}$ is a large fixed positive constant. Obviously, we set $\tau_{\varepsilon}=T_{\varepsilon}$ if none of the conditions is satisfied for all $t<T_{\varepsilon}$.

From the previous definition, it follows that $v=\mathcal{O}_{H_{\varepsilon}^{1}}\left(\varepsilon^{k-3-\tilde{\kappa}}\right)$, i.e.

$$
\sup _{t \in\left[0, T_{\varepsilon}\right]}\|v(t)\|_{H_{\varepsilon}^{1}} \leq C_{0} \varepsilon^{k-3-\tilde{\kappa}} .
$$

Hence, for this stopping time considered, the bound in $L^{2}$-norm provided by Theorem 3.5 (i.e., $\|v(t)\|<$ $C \varepsilon^{k-2}$ for all $t \leq \tau_{\varepsilon}$ with high probability, as long as $T_{\varepsilon}$ is a polynomial in $\varepsilon^{-1}$ ) is much stronger than the $L^{2}$-bound given in the $H_{\varepsilon}^{1}$-norm. 
For the remaining part of the argument we assume that we only consider times before the exit time, i.e $t \leq \tau_{\varepsilon}$ and we fix in the bound of $\mathcal{B} K \geq k$. We know by Theorems 2.7, 2.9 that

$$
\sup _{\left[0, \tau_{\varepsilon}\right]}\left|b-c \varepsilon^{2}\right| \leq C \varepsilon^{k-3 / 2} .
$$

Thus, relation 3.23 yields

$$
\begin{aligned}
d\|\nabla v\|^{2} \leq & -\varepsilon^{2}\|\Delta v\|^{2} d t+2 T_{2} \\
& +C\left(\varepsilon^{2 k-6}+\varepsilon^{-3} \eta_{0}^{2}+\varepsilon^{-2} \eta_{0}+\eta_{2}\right) d t \\
& +2(\mathcal{A}, d W)+2(\nabla v, \nabla d W) .
\end{aligned}
$$

We complete the estimation of the term $T_{2}$, by using the bound given by (3.22) and the following relations

$$
\|v\| \leq C \varepsilon^{k-2} \quad \text { and } \quad\|\nabla v\|^{2} \leq\|\Delta v\|\|v\| .
$$

Therefore, for $k \geq 3$, we obtain by Young's inequality

$$
\begin{aligned}
T_{2} & \leq\left(\|\nabla v\|^{2}+C \varepsilon^{-1}\|v\|\|\nabla v\|^{2}+C \varepsilon^{-1}\|v\|^{2}\|\nabla v\|+C \varepsilon^{-1}\|v\|\|\nabla v\|\right) d t \\
& \leq\left(4\|\nabla v\|^{2}+C \varepsilon^{2 k-6}\right) d t \\
& \leq\left(\frac{1}{4} \varepsilon^{2}\|\Delta v\|^{2}+C \varepsilon^{-2}\|v\|^{2}+C \varepsilon^{2 k-6}\right) d t \\
& \leq\left(\frac{1}{4} \varepsilon^{2}\|\Delta v\|^{2}+C \varepsilon^{2 k-6}\right) d t
\end{aligned}
$$

To close the argument, we need a Poincaré type estimate. More specifically, for any function satisfying Neumann boundary conditions there exists some positive constant $c>0$ such that

$$
2 c\|\nabla v\|^{2} \leq\|\Delta v\|^{2} .
$$

To prove the statement above, first let us denote by $\bar{v}$ the spatial average of $v$, and then use interpolation and the standard Poincaré inequality. So, we have

$$
\|\nabla v\|^{2}=\|\nabla(v-\bar{v})\|^{2} \leq\|v-\bar{v}\|\|\Delta(v-\bar{v})\| \leq C\|\nabla(v-\bar{v})\|\|\Delta(v-\bar{v})\|=C\|\nabla v\|\|\Delta v\| .
$$

Therefore, by 3.25 and 3.26 , we obtain the following lemma.

Lemma 3.9. If $k \geq 3$ and for times $t \leq \tau_{\varepsilon}$, with exit time $\tau_{\varepsilon}$ given by $(3.24)$, then with the constant $c>0$ appearing in Poincaré inequality (3.27) the following relation holds true

$$
d\|\nabla v\|^{2}+c \varepsilon^{2}\|\nabla v\|^{2} d t=\Gamma_{\varepsilon} d t+(Z, d W)+(\Psi, d \nabla W)
$$

for $\Gamma_{\varepsilon}, Z$ and $\Psi$ processes such that up to $\tau_{\varepsilon}$ the following bounds hold true

$$
\begin{gathered}
\left|\Gamma_{\varepsilon}\right| \leq C\left(\varepsilon^{2 k-6}+\varepsilon^{-3} \eta_{0}^{2}+\varepsilon^{-2} \eta_{0}+\eta_{2}\right), \\
\|Z\|^{2} \leq C \varepsilon^{-2}\|\nabla v\|^{2}, \quad \text { and } \quad\|\Psi\|^{2} \leq C\|\nabla v\|^{2} .
\end{gathered}
$$

Furthermore, it holds by integrating up to the stopping time that

$$
\mathbb{E}\left\|\nabla v\left(t \wedge \tau_{\varepsilon}\right)\right\|^{2}+c \varepsilon^{2} \mathbb{E} \int_{0}^{t \wedge \tau_{\varepsilon}}\|\nabla v(s)\|^{2} d s \leq\|\nabla v(0)\|^{2}+\Gamma_{\varepsilon} T_{\varepsilon} .
$$

We remark, that as in the $L^{2}$-case this lemma is only the first step for proving stability; higher moments will be derived by an induction argument using higher powers of $\|\nabla v\|^{2}$ in the following section. 
3.4. Long-Time $H^{1}$-stability. Keeping the same assumptions as in Lemma 3.9 we proceed similarily to the $L^{2}$-stability result by estimating for any integer $p>1$ the $p^{\text {th }}$-moment of $\|\nabla v\|^{2}$. First Itô calculus yields

$$
d\|\nabla v\|^{2 p}=p\|\nabla v\|^{2 p-2} d\|\nabla v\|^{2}+p(p-1)\|\nabla v\|^{2 p-4}\left[d\|\nabla v\|^{2}\right]^{2} .
$$

Using now (3.28), we obtain for the Itô-correction

$$
\left[d\|\nabla v\|^{2}\right]^{2}=(Z, \mathcal{Q} Z) d t+(\Psi, \Delta \mathcal{Q} \Psi) d t+2(Z, d W)(\Psi, d \nabla W) .
$$

By series expansion of $W$ and Cauchy-Schwarz inequality, we have

$$
\begin{aligned}
(Z, d W)(\psi, d \nabla W) & =\sum a_{i}^{2}\left(Z, e_{i}\right)\left(\psi, \nabla e_{i}\right) \leq \sum a_{i}^{2}\left\|e_{i}\right\|\left\|\nabla e_{i}\right\|\|Z\|\|\psi\| d t \\
& \leq\|Z\|\|\psi\| \sqrt{\eta_{0} \eta_{2}} d t \\
& \leq \frac{1}{2}\|Z\|^{2} \eta_{0} d t+\frac{1}{2}\|\psi\|^{2} \eta_{2} d t .
\end{aligned}
$$

Recall that as before the estimates are true in the integral from, and we only estimate the drift part. By relations (3.33) and (3.34), we arrive at the following bound for the Itô-correction

$$
\begin{aligned}
{\left[d\|\nabla v\|^{2}\right]^{2} } & \leq\left(\|\mathcal{Q}\|\|Z\|^{2}+\|\Psi\|^{2}\|\Delta \mathcal{Q}\|\right) d t+2\|Z\|\|\Psi\| \sqrt{\eta_{0} \eta_{2}} d t \\
& \leq C\left(\|Z\|^{2} \eta_{0}+\|\Psi\|^{2} \eta_{2}\right) d t .
\end{aligned}
$$

Replacing 3.35 in 3.32 and using (3.28), we get

$$
\begin{aligned}
d\|\nabla v\|^{2 p} \leq & p\|\nabla v\|^{2 p-2} d\|\nabla v\|^{2}+C p(p-1)\|\nabla v\|^{2 p-4}\left(\|Z\|^{2} \eta_{0}+\|\Psi\|^{2} \eta_{2}\right) d t \\
\leq & p\|\nabla v\|^{2 p-2}\left(\Gamma_{\varepsilon}-c \varepsilon^{2}\|\nabla v\|^{2}\right) d t \\
& +p\|\nabla v\|^{2 p-2}[(Z, d W)+(\Psi, d \nabla W)] \\
& +C p(p-1)\|\nabla v\|^{2 p-4}\left(\|Z\|^{2} \eta_{0}+\|\Psi\|^{2} \eta_{2}\right) d t .
\end{aligned}
$$

In the previous argument, we used (3.30) and the fact that $\|v\| \leq C \varepsilon^{k-2}$ up to $\tau_{\varepsilon}$. Hence, we obtain the following for all times $t \leq \tau_{\varepsilon}$, where again all appearing constants may depend on $p$.

$$
\begin{aligned}
d\|\nabla v\|^{2 p} \leq & p\|\nabla v\|^{2 p-2}\left(\Gamma_{\varepsilon}-c \varepsilon^{2}\|\nabla v\|^{2}\right) d t+p\|\nabla v\|^{2 p-2}[(Z, d W)+(\Psi, d \nabla W)] \\
& +C\|\nabla v\|^{2 p-2} \varepsilon^{-2} \eta_{0} d t+C\|\nabla v\|^{2 p-2} \eta_{2} d t .
\end{aligned}
$$

Integrating (3.36) up to the stopping time and taking expectations we derive the following lemma.

Lemma 3.10. Under the assumptions of Lemma 3.9 for any integer $p \geq 2$ the following estimate holds true

$$
\mathbb{E}\left\|\nabla v\left(\tau_{\varepsilon}\right)\right\|^{2 p}+c \varepsilon^{2} p \mathbb{E} \int_{0}^{\tau_{\varepsilon}}\|\nabla v(t)\|^{2 p} d t \leq\|\nabla v(0)\|^{2 p}+C\left[\Gamma_{\varepsilon}+\varepsilon^{-2} \eta_{0}+\eta_{2}\right] \cdot \mathbb{E} \int_{0}^{\tau_{\varepsilon}}\|\nabla v(t)\|^{2 p-2} d t
$$

where $c, C>0$ are constants that may depend on $p \in \mathbb{N}$ and $\Gamma_{\varepsilon}$ is defined by 3.29).

Keeping the same assumptions as those of Lemma 3.9, we consider a sufficiently small noise such that

$$
\varepsilon^{-2} \eta_{0}+\eta_{2} \leq C \varepsilon^{2 k-6}
$$

which implies that $\left|\Gamma_{\varepsilon}+\varepsilon^{-2} \eta_{0}+\eta_{2}\right| \leq C \varepsilon^{2 k-6}$. Furthermore, in (3.37) we define

$$
K_{p}:=\mathbb{E} \int_{0}^{\tau_{\varepsilon}}\|\nabla v(t)\|^{2 p} d t
$$

and thus, we obtain for $p \geq 2$

$$
\begin{aligned}
K_{p} & \leq \varepsilon^{-2}\|\nabla v(0)\|^{2 p}+C \varepsilon^{-2}\left[\Gamma_{\varepsilon}+\varepsilon^{-2} \eta_{0}+\eta_{2}\right] K_{p-1} \\
& \leq \varepsilon^{-2}\|\nabla v(0)\|^{2 p}+C \varepsilon^{2 k-8} K_{p-1} \\
& =\varepsilon^{-2} A^{p}+a K_{p-1},
\end{aligned}
$$


for

So, we have inductively

$$
A:=\|\nabla v(0)\|^{2}, \quad a:=C \varepsilon^{2 k-8}<1 .
$$

$$
\begin{aligned}
K_{p} \leq \varepsilon^{-2} A^{p}+a K_{p-1} & \leq \varepsilon^{-2} A^{p}+\varepsilon^{-2} a K_{p-2} A^{p-1}+2 a^{2} K_{p-2} \\
& \leq \ldots \leq C \varepsilon^{-2} \sum_{i=2}^{p} a^{p-i} A^{i}+C a^{p-1} K_{1} .
\end{aligned}
$$

By definition and (3.31) of Lemma 3.9 we derive for the final step

$$
K_{1} \leq \varepsilon^{-2} A+\varepsilon^{-2} \Gamma_{\varepsilon} T_{\varepsilon} \leq \varepsilon^{-2} A+C a T_{\varepsilon} .
$$

Hence, we obtain an overall bound on $K_{p}$ given by

$$
K_{p} \leq C \varepsilon^{-2} \sum_{i=1}^{p} a^{p-i} A^{i}+C a^{p} T_{\varepsilon} \leq C\left[\varepsilon^{-2}+T_{\varepsilon}\right] a^{p}+C \varepsilon^{-2} A^{p},
$$

for a constant $C>0$ depending on $p$. This yields the following lemma.

Lemma 3.11. Let $k \geq 3$ and consider the exit time $\tau_{\varepsilon}$ given by (3.24). If the noise is sufficiently small so that

$$
\Gamma_{\varepsilon}+\varepsilon^{-2} \eta_{0}+\eta_{2} \leq C \varepsilon^{2 k-6}
$$

and if the gradient of the initial condition is bounded by

$$
\|\nabla v(0)\|^{2} \leq a:=C \varepsilon^{2 k-8},
$$

then for any integer $p>1$ it holds that at the exit time the gradient of $v$ is bounded by

$$
\mathbb{E}\left\|\nabla v\left(\tau_{\varepsilon}\right)\right\|^{2 p} \leq C \varepsilon^{-2}\left[\varepsilon^{-2}+T_{\varepsilon}\right] a^{p} .
$$

Proof. Using the definitions of $a, A$, and $K_{p}$ and relation (3.37), we obtain

$$
\begin{aligned}
\mathbb{E}\left\|\nabla v\left(\tau_{\varepsilon}\right)\right\|^{2 p} & \leq A^{p}+C \varepsilon^{-2} a K_{p-1} \\
& \leq A^{p}+C \varepsilon^{-2}\left[\varepsilon^{-2}+T_{\varepsilon}\right] a^{p}+C \varepsilon^{-4} a A^{p-1} \\
& \leq C \varepsilon^{-2}\left[\varepsilon^{-2}+T_{\varepsilon}\right] a^{p} .
\end{aligned}
$$

Remark 3.12. Note that the previous Lemma only provides a bound on $\nabla v$ up to the exit time using the weaker condition $k \geq 3$, while the $L^{2}$-stability proven in the previous section is valid only for $k>5$.

We proceed now to the proof of the following main stability theorem.

Theorem 3.13. Consider the exit time

$$
\tau_{\varepsilon}:=\inf \left\{t \in\left[0, T_{\varepsilon}\right]:\|\nabla v(t)\|>C_{0} \varepsilon^{k-4-\tilde{\kappa}} \text { or }\|v(t)\|>C_{0} \varepsilon^{k-2}\right\},
$$

where $T_{\varepsilon}:=\varepsilon^{-N}$ for arbitrarily large but fixed $N>0$ and for arbitrarily small but fixed $\tilde{\kappa}>0$. Let also, for some fixed constant $\eta>0$

$$
k>5, \quad\|v(0)\|<\eta \varepsilon^{k-2} \text { and }\|\nabla v(0)\|<\eta \varepsilon^{k-4} .
$$

In addition, let the noise be sufficiently small so that

$$
\eta_{0} \leq C \varepsilon^{2 k-2+\tilde{k}} \text { and } \eta_{2} \leq C \varepsilon^{2 k-6},
$$

for some fixed $\tilde{k}>0$ small. Then the probability $\mathbb{P}\left(\tau_{\varepsilon}<T_{\varepsilon}\right)$ is smaller than any power of $\varepsilon$, as $\varepsilon \rightarrow 0^{+}$. So, for very large times and with high probability, the solution stays close to the manifold in the $H_{\varepsilon}^{1}$-norm. 
Proof. Obviously, by the definition of the stopping time we have

$$
\mathbb{P}\left(\tau_{\varepsilon}<T_{\varepsilon}\right) \leq \mathbb{P}\left(\left\|\nabla v\left(\tau_{\varepsilon}\right)\right\| \geq C_{0} \varepsilon^{k-4-\tilde{\kappa}}\right)+\mathbb{P}\left(\left\|v\left(\tau_{\varepsilon}\right)\right\| \geq C_{0} \varepsilon^{k-2}\right) .
$$

Using now Theorem 3.5 for any large $\ell>1$ together with Chebychev's inequality for arbitrarily large $p>1$, we obtain

$$
\begin{aligned}
\mathbb{P}\left(\tau_{\varepsilon}<T_{\varepsilon}\right) & \leq C \varepsilon^{-2 p(k-4-\tilde{\kappa})} \mathbb{E}\left\|\nabla v\left(\tau_{\varepsilon}\right)\right\|^{2 p}+C \varepsilon^{\ell} \\
& \leq C \varepsilon^{2 p \tilde{\kappa}} \varepsilon^{-2}\left[\varepsilon^{-2}+T_{\varepsilon}\right]+C \varepsilon^{\ell},
\end{aligned}
$$

where Lemma 3.11 was applied. Choosing $p \gg \frac{1}{2 \tilde{\kappa}}$ yields the result.

Let us rephrase Theorem 3.13 slightly:

Corollary 3.14. Under the assumptions of Theorem 3.13 and if $w(0) \in \mathcal{N}_{L^{2}}^{\eta \varepsilon^{k-2}}$ and $\nabla w(0) \in \mathcal{N}_{L^{2}}^{\eta \varepsilon^{k-4}}$ for any $\eta>0$, then for any sufficiently large $C>\eta$ and any $q \in \mathbb{N}$ there exists a constant $C_{q}>0$ such that

$$
\mathbb{P}\left(w(t) \in \mathcal{N}_{H_{\varepsilon}^{1}}^{C \varepsilon^{k-3-\tilde{k}}} \text { for all } t \in\left[0, \varepsilon^{-q}\right]\right) \geq 1-C_{q} \varepsilon^{q} .
$$

\section{Estimates}

In this section, we will present the estimates of $\left(\partial_{\xi}^{2} u, \partial_{\xi} u\right)$ and of $\partial_{\xi} u, \partial_{\xi}^{2} u, \partial_{\xi}^{3} u$ in various norms, used throughout the previous sections. Recall that $\Gamma$ was the small semicircle where $u^{\xi}=0$, and apart from a small neighborhood, $u^{\xi} \approx 1$ inside and $u^{\xi} \approx-1$ outside. Our proof extends certain lower order results of [2] derived for the deterministic problem. One key technical difference is that due to the Itô-correction in the Itô-formula, we need bounds on higher order derivatives of $u$.

First, avoiding the brute force estimate based on Cauchy-Schwarz inequality, we present a refined estimate for the scalar product between $\partial_{\xi}^{2} u$ and $\partial_{\xi} u$.

Theorem 4.1. There exists a constant $C>0$ such that

$$
\left|\left(\partial_{\xi}^{2} u, \partial_{\xi} u\right)\right| \leq C \varepsilon^{-1} .
$$

Proof. We may consider the case that $\Omega_{\delta}$ is a normal graph over the unit sphere, so that any $x \in \Omega_{\delta}$ is represented by $x=(r \cos (\theta), r \sin (\theta))$ for any $0 \leq \theta<2 \pi$ and any $0 \leq r<R(\theta)$, where $R(\theta)$ is the distance of the point of the boundary $\partial \Omega_{\delta}$ from the origin, at the angle $\theta$. This is not restrictive since, as we shall see, our integral vanishes outside a neighborhood of a point on $\partial \Omega_{\delta}$. The coordinate $r$ here should not be confused with the local coordinate near $\Gamma$. Therefore, we have

$$
\left(\partial_{\xi}^{2} u, \partial_{\xi} u\right)=\int_{\Omega_{\delta}} \partial_{\xi}^{2} u \partial_{\xi} u d x=\int_{0}^{2 \pi} \int_{0}^{R(\theta)} \frac{1}{2} \frac{d}{d \xi}\left(\partial_{\xi} u\right)^{2} r d r d \theta .
$$

Observe that if $\partial \Omega_{\delta}=(a(\theta), b(\theta))=(R(\theta) \cos (\theta), R(\theta) \sin (\theta))$ for $\theta \in[0,2 \pi]$ then, the arc-length parameter $\xi$ of $\partial \Omega_{\delta}$ is given by

$$
\xi(\theta)=\int_{0}^{\theta}\left(a^{\prime}(t)^{2}+b^{\prime}(t)^{2}\right)^{1 / 2} d t .
$$

Therefore, $\xi_{\theta}=\left(R^{\prime}(\theta)^{2}+R(\theta)^{2}\right)^{1 / 2}$ and thus

$$
d \xi=\xi_{\theta} d \theta=\left(R^{\prime}(\theta)^{2}+R(\theta)^{2}\right)^{1 / 2} d \theta .
$$


Setting $L:=\left|\partial \Omega_{\delta}\right|$ and using that the boundary is a closed curve, it follows for $\hat{R}(\xi)=R(\theta)$ that

$$
\begin{aligned}
\left(\partial_{\xi}^{2} u, \partial_{\xi}(u)\right)= & \int_{0}^{L} \int_{0}^{\hat{R}(\xi)} \frac{1}{2} \frac{d}{d \xi}\left(\partial_{\xi}(u)\right)^{2} r d r\left(R^{\prime}(\theta)^{2}+R(\theta)^{2}\right)^{-1 / 2} d \xi \\
= & \int_{0}^{L} \frac{1}{2} \frac{d}{d \xi}\left(\int_{0}^{\hat{R}(\xi)}\left(\partial_{\xi} u\right)^{2} r d r\right)\left(R^{\prime}(\theta)^{2}+R(\theta)^{2}\right)^{-1 / 2} d \xi \\
& -\int_{0}^{L} \frac{1}{2} \hat{R}_{\xi}(\xi)\left(\partial _ { \xi } ( u ) \left(\hat{R}(\xi)^{2} \hat{R}(\xi)\left(R^{\prime}(\theta)^{2}+R(\theta)^{2}\right)^{-1 / 2} d \xi\right.\right. \\
= & {\left[\frac{1}{2}\left(\int_{0}^{\hat{R}(\xi)}\left(\partial_{\xi} u\right)^{2} r d r\right)\left(R^{\prime}(\theta)^{2}+R(\theta)^{2}\right)^{-1 / 2}\right]_{0}^{L} } \\
& -\int_{0}^{L} \frac{1}{2}\left(\int_{0}^{\hat{R}(\xi)}\left(\partial_{\xi} u\right)^{2} r d r\right) \partial_{\xi}\left(\left(R^{\prime}(\theta)^{2}+R(\theta)^{2}\right)^{-1 / 2}\right) d \xi \\
& -\int_{0}^{L} \frac{1}{2} \hat{R}_{\xi}(\xi)\left(\partial _ { \xi } ( u ) \left(\hat{R}(\xi)^{2} \hat{R}(\xi)\left(R^{\prime}(\theta)^{2}+R(\theta)^{2}\right)^{-1 / 2} d \xi\right.\right. \\
= & -\int_{0}^{L} \frac{1}{2}\left(\int_{0}^{\hat{R}(\xi)}\left(\partial_{\xi} u\right)^{2} r d r\right) \partial_{\xi}\left(\left(R^{\prime}(\theta)^{2}+R(\theta)^{2}\right)^{-1 / 2}\right) d \xi \\
& -\int_{0}^{L} \frac{1}{2} \hat{R}_{\xi}(\xi)\left(\partial _ { \xi } ( u ) \left(\hat{R}(\xi)^{2} \hat{R}(\xi)\left(R^{\prime}(\theta)^{2}+R(\theta)^{2}\right)^{-1 / 2} d \xi .\right.\right.
\end{aligned}
$$

Note that the construction of $u$ in [2] shows that $\partial_{\xi} u$ vanishes outside a neighborhood of $\Gamma$ of width $2 \varepsilon \log ^{2} \varepsilon$, which allows us to use the representation of $\partial \Omega_{\delta}$.

Returning to the original set $\Omega=\delta \Omega_{\delta}$, note that the arc-length parameter of its boundary is $\tilde{\xi}=\delta \xi$. Moreover, we shall frequently use indices to denote derivatives, in order to stay close to the notation of [2].

If $\tilde{R}(\tilde{\xi})$ is the distance of the boundary $\partial \Omega$ from the origin, then we have that

$$
\tilde{R}(\tilde{\xi})=\delta \hat{R}(\xi), \quad \tilde{R}_{\tilde{\xi}}(\tilde{\xi}), \quad \text { and } \quad \tilde{R}_{\tilde{\xi} \tilde{\xi}}(\tilde{\xi}) \quad \text { are all independent of } \delta
$$

and thus, by simple rescaling arguments we obtain that

$$
\hat{R}_{\xi}(\xi) \text { and } \quad \delta^{-1} \hat{R}_{\xi \xi}(\xi) \text { are independent of } \delta .
$$

If now $\bar{R}(\theta)$ is the distance from the origin to the boundary $\partial \Omega$ at angle $\theta$, then we have that

$$
\begin{array}{r}
\hat{R}(\xi)=R(\theta), \quad \delta \hat{R}(\xi)=\tilde{R}(\tilde{\xi})=\bar{R}(\theta), \\
\bar{R}_{\theta}(\theta), \quad \text { and } \quad \bar{R}_{\theta \theta}(\theta) \text { are all independent of } \delta,
\end{array}
$$

while,

$$
R_{\theta}(\theta)=\delta^{-1} \bar{R}_{\theta}(\theta) \quad \text { and } \quad R_{\theta \theta}(\theta)=\delta^{-1} \bar{R}_{\theta \theta}(\theta) .
$$

But $R(\theta)=\hat{R}(\xi)$ and so, it follows that $R_{\theta}(\theta)=\hat{R}_{\xi}(\xi) \xi_{\theta}=\hat{R}_{\xi}(\xi)\left(R_{\theta}^{2}+R^{2}\right)^{1 / 2}$. Hence, we obtain

$$
\partial_{\xi}\left(\left(R^{\prime}(\theta)^{2}+R(\theta)^{2}\right)^{-1 / 2}\right)=\partial_{\xi}\left(\frac{\hat{R}_{\xi}(\xi)}{R_{\theta}(\theta)}\right)=\frac{\hat{R}_{\xi \xi} R_{\theta}-\hat{R}_{\xi} R_{\theta \theta} \theta_{\xi}}{R_{\theta}^{2}} .
$$

In $\Omega$, observe that

$$
\frac{\partial \theta}{\partial \tilde{\xi}} \text { is independent of } \delta, \text { and } \tilde{\xi}=\delta \xi
$$

So, we arrive at

$$
\left|\partial_{\xi}\left(\left(R^{\prime}(\theta)^{2}+R(\theta)^{2}\right)^{-1 / 2}\right)\right| \leq C \delta^{2} .
$$


But (cf. 2], p. 294) in a neighborhood of $\Gamma$ of width $\varepsilon \log ^{2} \varepsilon$, using the local coordinates $(r, s)$ with $r$ being the signed distance from $\Gamma$ and $s$ being the arc-length parameter of $\Gamma$, we obtain

$$
\partial_{\xi} u=-\frac{1}{\varepsilon}\left\{\cos (\pi s /|\Gamma|) \dot{U}\left(\frac{r}{\varepsilon}\right)+\mathcal{A}\right\}
$$

where $|\mathcal{A}| \leq C \varepsilon$, and $U$ is the heteroclinic solution to the one-dimensional problem connecting \pm 1 , given by (1.7).

The smooth cut-off function maintains this estimate on the support of $\partial_{\xi} u$, being a neighborhood of $\Gamma$ of width $2 \varepsilon \log ^{2} \varepsilon$. Furthermore, $\dot{U}$ decays exponentially, as shown in the classical work of Fife and McLeod 22]. So, we obtain

$$
\begin{aligned}
\left|\left(\partial_{\xi}^{2} u, \partial_{\xi} u\right)\right| \leq & \int_{0}^{L} \frac{1}{2}\left(\int_{0}^{\hat{R}(\xi)}\left|\partial_{\xi}(u)\right|^{2} r d r\right)\left|\partial_{\xi}\left(\left(R^{\prime}(\theta)^{2}+R(\theta)^{2}\right)^{-1 / 2}\right)\right| d \xi \\
& +\int_{0}^{L} \frac{1}{2}\left|\hat{R}_{\xi}(\xi)\right|\left|\partial_{\xi}(u)(\hat{R}, \xi)\right|^{2} \hat{R}(\xi)\left(R^{\prime}(\theta)^{2}+R(\theta)^{2}\right)^{-1 / 2} d \xi \\
\leq & C \varepsilon^{-1} \delta^{2}+C \varepsilon^{-1} \leq C \varepsilon^{-1}
\end{aligned}
$$

We now prove certain estimates for various derivatives of $u$ with respect to $\xi$. Some of these results are already proven in [2] but are included here for completeness of presentation.

Theorem 4.2. It holds for some universal constant $C>0$ that

$$
\begin{array}{cl}
\left\|\partial_{\xi} u\right\|_{L^{1}} \leq C, & \left\|\partial_{\xi} u\right\| \leq C \varepsilon^{-\frac{1}{2}}, \\
\left\|\partial_{\xi}^{2} u\right\| \leq C \varepsilon^{-\frac{3}{2}}, \quad \text { and } \quad\left\|\partial_{\xi}^{3} u\right\| \leq C \varepsilon^{-\frac{5}{2}} .
\end{array}
$$

Proof. First recall the representation of $\partial_{\xi} u$ (4.2), which we will use later in the proof. The function $u$ is the sum of two terms written in local coordinates, an interior expansion $u^{I}$ and a corner layer expansion $u^{B \pm}$ :

$$
u=u^{I}+u^{B}
$$

where (from [2] p. 251, 254)

$$
u^{I}=U(R)+\varepsilon \sum_{j \geq 0} \varepsilon^{j} u_{j}^{I}(R, \cdots),
$$

and

$$
u^{B}=\sum_{j \geq 1} \varepsilon^{j} u_{j}^{B \pm}(R, \cdots) .
$$

Let $D_{a}^{k}$ denote the $k$ partial derivative with respect to variable $a$. For the interior layer expansion it holds that (see 2] p. 252)

$$
\left|D_{R}^{m} D_{s}^{n} D_{\xi}^{l} u_{j}^{I}\right| \leq C
$$

uniformly in $\varepsilon$ and for any integer $m, n, l \geq 0$. Since the interior expansion in local coordinates has a smooth extension to the whole domain (by 4.5, this means that $u_{j}^{I}$ have smooth extensions to the whole domain), then 4.7, which is true for $u_{j}^{I}$ in local coordinates, is true for their smooth extensions too. On the other hand for a given $\xi$, the construction of $u^{B \pm}$ in local coordinates (see [2] p. 241) permits as to derive that $D_{R}^{m} D_{s}^{n} D_{\xi}^{l} u_{j}^{B \pm}$ are bounded uniformly in $\varepsilon$, i.e.,

$$
\left|D_{R}^{m} D_{s}^{n} D_{\xi}^{l} u_{j}^{B \pm}\right| \leq C,
$$

for any integer $m, n, l \geq 0$. 
Furthermore, $u$ is smoothly extended to the whole domain, cf. p. 257 of [2], and thus, is given as

$$
u=\tilde{u}^{I}+\tilde{u}^{B}
$$

for $\tilde{u}^{I}$ and $\tilde{u}^{B}$ suitable modifications of $u^{I}$ and $u^{B}$ respectively. More specifically, for the cut-off function $\zeta \in C^{\infty}$ with $\zeta(s)=1$ if $s>1, \zeta(s)=0$ if $s<0$ and $s \zeta^{\prime}(s)>0$ on $\mathbb{R}, \tilde{u}^{I}$ is given as

$$
\begin{aligned}
\tilde{u}^{I} & :=\left(1-\zeta^{+}-\zeta^{-}\right) \cdot\left(U(R)+\varepsilon \sum_{j \geq 0} \varepsilon^{j} u_{j}^{I}(R, \cdots)\right)+\zeta^{+} u^{+}(\xi)+\zeta^{-} u^{-}(\xi) \\
& =\left(1-\zeta^{+}-\zeta^{-}\right) u^{I}+\zeta^{+} u^{+}(\xi)+\zeta^{-} u^{-}(\xi),
\end{aligned}
$$

where

$$
\zeta^{ \pm}=\zeta\left( \pm \frac{r(\xi, \cdots)}{\varepsilon \ln \varepsilon^{2}}-1\right) \quad \text { and } \quad u^{ \pm}= \pm 1+\varepsilon \sum_{j \geq 0} \varepsilon^{j} u_{j}^{ \pm} .
$$

Note that $u_{j}^{ \pm}$are smooth and uniformly bounded in $\varepsilon$, and the same holds for their derivatives of any order. Easily, by taking the derivative in $\xi$ we obtain

$$
\begin{aligned}
\partial_{\xi} \tilde{u}^{I} & =\partial_{\xi}\left(1-\zeta^{+}-\zeta^{-}\right) \cdot u^{I}+\left(1-\zeta^{+}-\zeta^{-}\right) \cdot \partial_{\xi} u^{I}+\partial_{\xi} \zeta^{+} \cdot u^{+}+\zeta^{+} \cdot \partial_{\xi} u^{+}+\partial_{\xi} \zeta^{-} \cdot u^{-}+\zeta^{-} \cdot \partial_{\xi} u^{-} \\
& \leq C\left[\frac{1}{\varepsilon \ln \varepsilon^{2}}\left|u^{I}\right|+\frac{1}{\varepsilon}\left|u^{I}\right|+\frac{1}{\varepsilon \ln \varepsilon^{2}}+\left|u^{I}\right|\right] \leq C\left|\partial_{\xi} u^{I}\right|
\end{aligned}
$$

where we used that $\frac{1}{\varepsilon}\left|u^{I}\right| \leq C\left|\partial_{\xi} u^{I}\right|$ (to be proved in the sequel). So, to estimate $\partial_{\xi} \tilde{u}^{I}$ we need to estimate $\partial_{\xi} u^{I}$, while by induction the same is possible for the derivatives of higher order i.e., we shall estimate $\partial_{\xi}^{m} \tilde{u}^{I}$ by estimating $\partial_{\xi}^{m} u^{I}$ for $m=1,2,3$. In the sequel, we derive the estimate of $\partial_{\xi}^{m} u^{I}$ in detail.

An analogous construction for $\tilde{u}^{B}$ gives that $\partial_{\xi}^{m} \tilde{u}^{B} \leq C\left|\partial_{\xi}^{m} u^{B}\right|$, so it is sufficient to estimate $\partial_{\xi}^{m} u^{B}$ for $m=1,2,3$.

In addition, $D_{\xi}^{l} s$ and $D_{\xi}^{l} r$ exist for $l \geq 0$ and are uniformly bounded for any $\varepsilon$ (for convenience we refer to [2] for the precise formulas which we abbreviate here only as $r_{\xi}=-\cos (\pi s /|\Gamma|)+\mathcal{O}(\delta)$ and and $\left.s_{\xi}=\mathcal{O}(\varepsilon+h+|r|)\right)$ so,

$$
\left|D_{\xi}^{l} s\right|<C \text { and }\left|D_{\xi}^{l} r\right|<C
$$

for any $l \geq 0$ uniformly in $\varepsilon$.

But, cf. 2] p. 258 states

$$
\frac{\partial x}{\partial(R, s)}=\varepsilon(1+\varepsilon R \mathcal{K}),
$$

and thus for any compactly supported $g=g(R, \cdots)$ it holds that (as in p. 258 of [2])

$$
\int_{\Omega_{\delta}} g(R, \cdots) d x=\varepsilon \int_{-\infty}^{+\infty} d R \int_{S^{-}(R, \xi, \varepsilon)}^{S^{+}(R, \xi, \varepsilon)}(1+\varepsilon R \mathcal{K}) g(R, \cdots) d s
$$

for the resulting $S^{-}$and $S^{+}$, where the change of variables from local to global coordinates is valid. Since $\Omega_{\delta}$ is bounded and $R=\frac{r}{\varepsilon}$ then if $g(R, \cdots)$ is bounded uniformly for any $\varepsilon$, for any $R \in \mathbb{R}$, we obtain

$$
\left|\int_{\Omega_{\delta}} g(R, \cdots) d x\right| \leq C
$$

uniformly in $\varepsilon$.

Using the above considerations, we are able to derive the estimates for the norms $\left\|\partial_{\xi} u\right\|_{L^{1}},\left\|\partial_{\xi} u\right\|,\left\|\partial_{\xi}^{2} u\right\|$ and $\left\|\partial_{\xi}^{3} u\right\|$, where $\partial_{\xi} u=\frac{\partial}{\partial \xi} u(r, s, \xi, \varepsilon)$, where we recall that both $r$ and $s$ depend on $\xi$. 
By using 4.2 we obtain first for the $L^{1}$-norm

$$
\begin{aligned}
\int_{\Omega_{\delta}}\left|\partial_{\xi} u\right| d x \leq C & \cdot\left(\varepsilon \int_{-\infty}^{+\infty} d R \int_{S^{-}(R, \xi, \varepsilon)}^{S^{+}(R, \xi, \varepsilon)}(1+\varepsilon R \mathcal{K})\left|\partial_{\xi} u\right| d s\right) \\
\leq C & \cdot\left(\varepsilon \int_{-\infty}^{+\infty} d R \int_{S^{-}(R, \xi, \varepsilon)}^{S^{+}(R, \xi, \varepsilon)}(1+\varepsilon R \mathcal{K}) \frac{1}{\varepsilon}|\cos (\pi s /|\Gamma|)||\dot{U}(R)| d s\right) \\
& +C \cdot\left(\varepsilon \int_{a}^{b} d R \int_{S^{-}(R, \xi, \varepsilon)}^{S^{+}(R, \xi, \varepsilon)}(1+\varepsilon R \mathcal{K}) \frac{1}{\varepsilon} \varepsilon d s\right) \leq C
\end{aligned}
$$

uniformly in $\varepsilon$, i.e., we proved the first estimate in 4.3 . Here, we used that

$$
\int_{-\infty}^{+\infty}\left|S^{+}(R, \xi, \varepsilon)-S^{-}(R, \xi, \varepsilon)\right||\dot{U}(R)| d R
$$

is bounded uniformly in $\varepsilon$, and we estimated $\partial_{\xi} u$ by using its local representation $\partial_{\xi}\left(u^{I}+u^{B}\right)$ which is estimated in relation 4.2 .

Furthermore, for the $L^{2}$-norm it follows that

$$
\begin{aligned}
\int_{\Omega_{\delta}}\left|\partial_{\xi} u\right|^{2} d x & \leq C \cdot\left(\varepsilon \int_{-\infty}^{+\infty} d R \int_{S^{-}(R, \xi, \varepsilon)}^{S^{+}(R, \xi, \varepsilon)}(1+\varepsilon R \mathcal{K})\left|\partial_{\xi} u\right|^{2} d s\right) \\
& \leq C \cdot\left(\varepsilon \int_{-\infty}^{+\infty} d R \int_{S^{-}(R, \xi, \varepsilon)}^{S^{+}(R, \xi, \varepsilon)}(1+\varepsilon R \mathcal{K}) \frac{1}{\varepsilon^{2}}|\cos (\pi s /|\Gamma|)|^{2}|\dot{U}(R)|^{2} d s+C\right) \leq C \varepsilon^{-1}
\end{aligned}
$$

so, we get the second equality in 4.3 . Here, we used that $\int_{-\infty}^{+\infty}\left|S^{+}(R, \xi, \varepsilon)-S^{-}(R, \xi, \varepsilon) \| \dot{U}(R)\right|^{2} d R$ is bounded uniformly in $\varepsilon$.

The second derivative of $u$ is more involved. Obviously, since

$$
\partial_{\xi} u=u_{r} r_{\xi}+u_{s} s_{\xi}+u_{\xi},
$$

it holds that

$$
\begin{aligned}
\partial_{\xi}^{2} u= & \left(u_{r r} r_{\xi}+u_{r s} s_{\xi}+u_{r \xi} \xi_{\xi}\right) r_{\xi}+u_{r} r_{\xi \xi} \\
& +\left(u_{s r} r_{\xi}+u_{s s} s_{\xi}+u_{s \xi} \xi_{\xi}\right) s_{\xi}+u_{s} s_{\xi \xi} \\
& +u_{\xi r} r_{\xi}+u_{\xi s} s_{\xi}+u_{\xi \xi} \xi_{\xi} .
\end{aligned}
$$

Hence, the worst term is $u_{r r}$ multiplied by a uniformly (in $\varepsilon$ ) bounded quantity. More specifically, using 4.5, 4.6), 4.7), 4.8 and 4.10, we observe that any derivative in $r$ gives $\varepsilon^{-1}$ since $R=\frac{r}{\varepsilon}$, while all the other derivatives of $s, r$ are uniformly bounded in $\varepsilon$ and the same is true for the derivatives of $u$ in $s, \xi$. Furthermore, considering the term $\partial_{\xi}^{3}(u)$, the chain rule analogously gives that the worst order is given by $u_{r r r}$.

In details, 4.5 and 4.7) give for $m, n, l \geq 0$

$$
\begin{aligned}
D_{r}^{m} D_{s}^{n} D_{\xi}^{l} u^{I} & =D_{r} U(R)+\varepsilon \varepsilon^{-m} \sum_{j \geq 0} \varepsilon^{j} D_{R}^{m} D_{s}^{n} D_{\xi}^{l} u_{j}^{I} \\
& \leq \varepsilon^{-m} D_{R}^{m} U(R)+C \varepsilon \varepsilon^{-m}
\end{aligned}
$$

while by 4.6 and 4.8 we obtain

$$
\begin{aligned}
D_{r}^{m} D_{s}^{n} D_{\xi}^{l} u^{B} & =\varepsilon^{-m} \sum_{j \geq 1} \varepsilon^{j} D_{R}^{m} D_{s}^{n} D_{\xi}^{l} u_{j}^{B \pm} \\
& \leq C \varepsilon^{-m} \sum_{j \geq 1} \varepsilon^{j} \leq C \varepsilon^{-m} \varepsilon .
\end{aligned}
$$


Since $u_{r r}=u_{r r}^{I}+u_{r r}^{B}$, then taking $m=2$ we get using 4.12 and 4.13), and the regularity of the second derivative of the heteroclinic,

$$
\begin{aligned}
\int_{\Omega_{\delta}}\left|\partial_{\xi}^{2} u\right|^{2} d x & \leq C \varepsilon \int_{-\infty}^{+\infty} d R \int_{S^{-}}^{S^{+}}(1+\varepsilon R \mathcal{K})\left|u_{r r}^{I}\right|^{2} d s+C \varepsilon \int_{-\infty}^{+\infty} d R \int_{S^{-}}^{S^{+}}(1+\varepsilon R \mathcal{K})\left|u_{r r}^{B}\right|^{2} d s \\
& \leq C \varepsilon \int_{-\infty}^{+\infty} \varepsilon^{-2 \cdot 2} d R \int_{S^{-}}^{S^{+}}(1+\varepsilon R \mathcal{K})\left|D_{R}^{2} U(R)\right|^{2} d s+C \varepsilon^{2} \varepsilon^{-2 \cdot 2}+C \varepsilon^{-2 \cdot 2} \varepsilon^{2} \\
& \leq C \varepsilon^{-3} .
\end{aligned}
$$

Thus, in accordance to 2 p. 297, we proved the first estimate in (4.4). For the previous relation we used that $\int_{-\infty}^{+\infty}\left|S^{+}(R, \xi, \varepsilon)-S^{-}(R, \xi, \varepsilon)\right|\left|D_{R}^{2} U(R)\right|^{2} d R$ is bounded uniformly in $\varepsilon$.

For the final term in (4.4) we can preform the analogous computation by taking $m=3$ in 4.12 and (4.13), since the third derivative of the heteroclinic is regular, this yields

$$
\begin{aligned}
\int_{\Omega_{\delta}}\left|\partial_{\xi}^{3} u\right|^{2} d x & \leq C \varepsilon \int_{-\infty}^{+\infty} d R \int_{S^{-}}^{S^{+}}(1+\varepsilon R \mathcal{K})\left|u_{r r r}^{I}\right|^{2} d s+C \varepsilon \int_{-\infty}^{+\infty} d R \int_{S^{-}}^{S^{+}}(1+\varepsilon R \mathcal{K})\left|u_{r r r}^{B}\right|^{2} d s \\
& \leq C \varepsilon \int_{-\infty}^{+\infty} \varepsilon^{-2 \cdot 3} d R \int_{S^{-}}^{S^{+}}(1+\varepsilon R \mathcal{K})\left|D_{R}^{3} U(R)\right|^{2} d s+C \varepsilon^{2} \varepsilon^{-2 \cdot 3}+C \varepsilon^{-2 \cdot 3} \varepsilon^{2} \leq C \varepsilon^{-5} .
\end{aligned}
$$

Thus, we proved the second inequality in (4.4). Here, we used that $\int_{-\infty}^{+\infty}\left|S^{+}(R, \xi, \varepsilon)-S^{-}(R, \xi, \varepsilon)\right|\left|D_{R}^{3} U(R)\right|^{2} d R$ is bounded uniformly in $\varepsilon$.

Remark 4.3. We note that by [2, p. 297] it holds also that $\left\|\partial_{\xi} u\right\| \geq C \varepsilon^{-\frac{1}{2}}$. Thus, there exist constants $0<C_{1} \leq C_{2}$ such that

$$
C_{1} \varepsilon^{-\frac{1}{2}} \leq\left\|\partial_{\xi} u\right\| \leq C_{2} \varepsilon^{-\frac{1}{2}} .
$$

Remark 4.4. Here, in our proof we used the regularity of the heteroclinic $U(R)$ up to derivatives of third order. This is provided in [22].

Remark 4.5. From the proof of Theorem 4.2 it is obvious that for any $k \geq 1$,

$$
\left\|\partial_{x}^{k} u\right\| \text { and }\left\|\partial_{\xi}^{k} u\right\|,
$$

have bounds of the same order in $\varepsilon$, and the same is true for the pair

$$
\left\|\partial_{x}^{k} u\right\|_{\infty} \quad \text { and }\left\|\partial_{\xi}^{k} u\right\|_{\infty} .
$$

Furthermore, by 2 , it holds that

$$
\left\|\partial_{\xi} u\right\|_{\infty} \leq C \varepsilon^{-1} \quad \text { and thus } \quad\|\nabla u\|_{\infty} \leq C \varepsilon^{-1}
$$

for some constants $C>0$.

Acknowledgment. The authors would like to thank the two anonymous referees for their valuable comments.

\section{REFERENCES}

[1] N. D. Alikakos, P. W. Bates, X. Chen, Convergence of the Cahn-Hilliard Equation to the Hele-Shaw Model, Arch. Rat. Mech. Anal., 128 (1994), pp. 165-205.

[2] N. D. Alikakos, X. Chen, G. Fusco, Motion of a droplet by surface tension along the boundary, Cal. Var., 11 (2000), pp. 233-305.

[3] S. Allen, J. W. CAhn, A microscopic theory for antiphase boundary motion and its application to antiphase domain coarsening, Acta. Metall., 27 (1979), pp. 1084-1095.

[4] D. C. Antonopoulou, D. Blömker, G. D. Karali, Front motion in the one-dimensional stochastic Cahn-Hilliard equation, SIAM J. Math. Anal., 44-5 (2012), pp. 3242-3280.

[5] P. W. Bates, P. Fife, The Dynamics of Nucleation for the Cahn-Hilliard equation, SIAM J. Appl. Math. 53 (1993), pp. 990-1008. 
[6] P. W. Bates, J. Jin, Global Dynamics of Boundary Droplets, Discrete and Continuous Dynamical Systems - Series A, 34 (2014), pp. 1-17.

[7] P. W. Bates, J. Xun, Metastable Patterns for the Cahn-Hilliard Equation, Part I, J. Differential Equations, 111 (1994), pp. 421-457.

[8] P. W. Bates, J. Xun, Metastable Patterns for the Cahn-Hilliard Equation: Part II. Layer Dynamics and Slow Invariant Manifold, J. Differential Equations, 117 (1995), pp. 165-216.

[9] N. Berglund, S. DutercQ, Interface dynamics of a metastable mass-conserving spatially extended diffusion, Preprint, (2015), http://arxiv:1508.04247v1

[10] N. Berglund, B. Gentz, Noise-Induced Phenomena in Slow-Fast Dynamical Systems. A Sample-Paths Approach, Probability and its Applications, Springer, (2006).

[11] D. Blömker, B. Gawron, and T. Wanner, Nucleation in the one-dimensional stochastic Cahn-Hilliard model, Discrete and Continuous Dynamical Systems, Series A, 27(1) (2010), pp. 25-52.

[12] D. Blömker, S. Maier-PaApe, T. Wanner, Spinodal decomposition for the Cahn-Hilliard-Cook equation, Comm. Math. Phys., 223(3) (2001), pp. 553-582.

[13] A. J. BraY, Domain Growth and Coarseninig, pp. 405-436 of "Phase Transitions and Relaxation in Systems with Competing Energy Scales: Proceedings of the NATO Advanced Study Institute," Geilo, Norway, April 13-23, 1993, (Nato Science Series C), Eds. T. Riste, David Sherrington.

[14] J. W. CAhn, J. E. Hilliard, Free energy of a nonuniform system. III. Nucleation in a two-component incompressible fluid, J. Chem. Phys. , 31 (1959),pp. 688-699.

[15] J. Carr, R. L. Pego, Metastable patterns in solutions of $u_{t}=\varepsilon^{2} u_{x x}-f(u)$, Communications on Pure and Applied Mathematics, 42(59, (1989), pp. 523-576.

[16] J. Carr, M. Gurtin, M. Slemrod, Structured phase transitions on a finite interval, Arch. Rat. Mech. Anal., 86 (1984), pp. 317-351.

[17] Xinfu Chen, M. Kowalczyk, Existence of equilibria for the Cahn-Hilliard equation via local minimizers of perimeter, Comm. PDE, 21 (1996), pp. 1207-1233.

[18] G. Da Prato, J. Zabczyk, Stochastic equations in infinite dimensions, 2nd Edition, Cambridge University Press, Cambridge 2014.

[19] J. P. Desi, H. Edrees, J. J. Price, E. Sander, T. Wanner, The dynamics of nucleation in stochastic Cahn-Morral systems, SIAM Journal on Applied Dynamical Systems, 10(2) (2011), pp. 707-743.

[20] P. C. Fife, Models For Phase Separation and Their Mathematics, Electron. J. Diff. Eqns., 2000(48) (2000), pp. 1-26.

[21] P. C. FIFE, Dynamics of internal layers and diffusive interfaces, CBMS-NSF 53, SIAM, 1988.

[22] P. C. FIFE, J. B. MCLEOD, The approach of solutions of nonlinear diffusion equations to travelling front solutions, Arch. Rat. Mech. Anal., 65 (1977), pp. 335-361.

[23] G. Fusco, J. K. Hale, Slow-motion manifolds, dormant instability, and singular perturbations, Journal of Dynamics and Differential Equations, 1(1), (1989), pp. 75-94.

[24] M. Hairer, M. D. Ryser, H. Weber, Triviality of the 2D stochastic Allen-Cahn equation, Electr. Journ. Probab., 17(39) (2012), pp. 1-14.

[25] M. Hairer, H. Weber, Large deviations for white-noise driven, nonlinear stochastic PDEs in two and three dimensions, Annales de la Faculté des Sciences de Toulouse, 24(1), (2015), 55-92.

[26] P. C. Hohenberg, B. I. Halperin, Theory of dynamic critical phenomena, Rev. mod. Phys., 49 (1977), pp. $435-479$.

[27] I. M. Lifshitz and V. V. Slyozov, The kinetics of precipitation from supersaturated solid solutions, J. Phys. Chem. Solids, 19 (1961), pp. 35-50.

[28] L. ModicA, Gradient theory of phase transition and singular perturbation, Arc. Rat. Mech. Anal., 107 (1989), pp. $123-142$.

[29] L. Modica, S. Mortola, Un esempio di Г-Convergenza, Boll. Un. Mat. Ital. B, 14 (1977), pp. $285-299$.

[30] F. Отto, H. Weber, M.G. Westdickenberg, Invariant measure of the stochastic Allen-Cahn equation: the regime of small noise and large system size, Electron. J. Probab., 19 (2014), pp. 1-76.

[31] N. C. Owen, P. Stenberg, Gradient flow and front propagation with boundary condact energy, Proc. Roy.Soc. Lond. Ser A, 437 (1992), pp. 715-728.

[32] M. RöGer, H. Weber, Tightness for a stochastic Allen-Cahn equation, Stochastic Partial Differential Equations: Analysis and Computations, 1(1) (2013), pp. 175-203.

[33] J. Rubistein, P. Sternberg, Nonlocal Reaction-Diffusion Equations and nucleation, IMA Journal of Applied Mathematics, 48 (1992), pp. 249-264.

[34] P. Stenberg, The effect of a singular perturbation on non-convex variational problems, Arc. Rat. Mech. Anal., 101 (1988), pp. 209-260.

[35] C. WAGneR, Theorie der Alterung von Niederschlägen durch Umlösen (Ostwald-Reifung), Z. Elektro chem., 65 (1961), pp. 581-594.

[36] H. WeBer, Sharp interface limit for invariant measures of a stochastic Allen-Cahn equation, Comm. Pure Appl. Math., 63(8) (2010), pp. 1071-1109. 
[37] S. Weber, The sharp interface limit of the stochastic Allen-Cahn equation in one space-dimension, University of Warwick, PhD thesis, 2015.

[38] N. K. YIP, Stochastic motion by mean curvature, Arch. Rational Mech. Anal., 144 (1998), pp. 313-355. 